\title{
Quantum fluid effects and parametric instabilities in microcavities
}

\author{
Cristiano Ciut:* \\ Laboratoire Pierre Aigrain, École Normale Supérieure, 24 rue Lhomond, 75005 Paris, France \\ Iacopo Carusotto \\ CRS BEC-INFM and Dipartimento di Fisica, Università di Trento, I-38050 Povo, Italy
}

(Dated: September 19, 2018)

\begin{abstract}
We present a description of the non-equilibrium properties of a microcavity polariton fluid, injected by a nearly-resonant continuous wave pump laser. In the first part, we point out the interplay between the peculiar dispersion of the Bogolubov-like polariton excitations and the onset of polariton parametric instabilities. We show how collective excitation spectra having no counterpart in equilibrium systems can be observed by tuning the excitation angle and frequency. In the second part, we explain the impact of these collective excitations on the in-plane propagation of the polariton fluid. We show that the resonant Rayleigh scattering induced by artificial or natural defects is a very sensitive tool to show fascinating effects such as polariton superfluidity or polariton Cherenkov effect. We present a comprehensive set of predicted far-field and near-field images for the resonant Rayleigh scattering emission.
\end{abstract}

\section{PREFACE}

In the last decade, the research group of Professor Marc Ilegems at EPFL has been working intensively and enthusiastically on the physics and device applications of artificial photonic systems, such as semiconductor microcavities and photonic crystals. In this Festschrift paper, we are going to present a theory of some exotic physical properties of coherently excited semiconductor microstructures in the strong exciton-photon coupling regime. We hope that the rich phenomenology here described will contribute to a very pleasant celebration of his 65 th birthday.

\section{INTRODUCTION}

The behavior of a quantum fluid has played an important role in many fields of condensed matter and atomic physics, ranging from superconductors to Helium fluids 1] and, during the last decade, Bose-Einstein condensates of cold trapped atoms [2]. One of the most dramatic manifestations of macroscopic coherence of an interacting many-body system is superfluidity 3].

In this paper, we will provide a comprehensive theoretical analysis of the predicted non-equilibrium propagation properties of a two-dimensional gas of polaritons in a semiconductor microcavity in the strong excitonphoton coupling regime [4, 5]. Thanks to their photonic component, polaritons can be coherently excited by an applied laser field and detected through the emitted light. Thanks to their excitonic component, polaritons have strong binary interactions, which have been shown to produce spectacular and rich polariton amplification effects through matter-wave stimulated collisions

*Electronic address: ciuti@lpa.ens.fr
6, 7, 8, 9, 10, 11], as well as spontaneous parametric instabilities [12, 13, 14, 15, 16, 17, 18]. Recently, a significant amount of research has been also focusing on the quantum optical properties of the polariton emission in the parametric regime with the possibility of observing polariton squeezing and polariton pair entanglement [19, 20, 21, 22, 23, 24, 25, 26].

Here, we are going to present a detailed discussion of the interplay between the peculiar polariton collective excitations and the rich variety of parametric instabilities, which occur in presence of a nearly-resonant continuous wave pump laser. In addition, we are going to discuss the impact on the propagation properties of a moving polariton fluid and analyze in detail the superfluid regime, which we predicted in a very recent Letter 27]. As our system is a strongly non-equilibrium one, the polariton field oscillation frequency is not fixed by any equation of state relating the chemical potential to the polariton density, but it can be tuned by the frequency of the exciting pump laser. This opens the possibility of having a collective excitation spectrum which has no counterpart in usual systems close to thermal equilibrium. In particular, we will analyze the propagation in presence of a static potential (either for the photonic or exciton component), which is known to produce resonant Rayleigh scattering (RRS) of the exciting laser field [14, 28, 29, 30]. Superfluidity of the polariton fluid manifests itself as a dramatic collapse of the resonant Rayleigh scattering intensity when the flow velocity imprinted by the exciting laser beam is slower than the interaction-induced sound velocity in the polariton fluid. Furthermore, a dramatic reshaping of the RRS pattern due to polariton-polariton interactions can be observed in both momentum and real space even at higher flow velocities, e.g. with the appearance of Cherenkov-like patterns. We will present a rich set of predicted far-field and near-field images of the resonant Rayleigh scattering emission. 


\section{HAMILTONIAN AND POLARITON MEAN-FIELD EQUATIONS}

In order to describe a planar microcavity containing a quantum well with an excitonic resonance strongly coupled to a cavity mode, we will consider the following model Hamiltonian [31]:

$$
\begin{array}{r}
\mathcal{H}=\int d \mathbf{x} \sum_{i j=\{X, C\}} \hat{\Psi}_{i}^{\dagger}(\mathbf{x})\left[\mathbf{h}_{i j}^{0}+V_{i}(\mathbf{x}) \delta_{i j}\right] \hat{\Psi}_{j}(\mathbf{x}) \\
+\frac{\hbar g}{2} \int d \mathbf{x} \hat{\Psi}_{X}^{\dagger}(\mathbf{x}) \hat{\Psi}_{X}^{\dagger}(\mathbf{x}) \hat{\Psi}_{X}(\mathbf{x}) \hat{\Psi}_{X}(\mathbf{x})+ \\
+\int d \mathbf{x} \hbar F_{p}(\mathbf{x}, t) \hat{\Psi}_{C}^{\dagger}(\mathbf{x})+\text { h.c. },
\end{array}
$$

where $\mathbf{x}$ is the in-plane spatial position and the field operators $\Psi_{X, C}(\mathbf{x})$ respectively describe excitons $(X)$ and cavity photons $(C)$. They satisfy Bose commutation rules, $\left[\hat{\Psi}_{i}(\mathbf{x}), \hat{\Psi}_{j}^{\dagger}\left(\mathbf{x}^{\prime}\right)\right]=\delta^{2}\left(\mathbf{x}-\mathbf{x}^{\prime}\right) \delta_{i j}$. Note that, for simplicity, we will limit our treatment to the case of polariton modes with the same circular polarization, which can be excited by a circularly polarized pump. The approach here presented can be generalized to the spin-dependent case by considering appropriate spin-dependent excitonexciton collisional potentials.

The single-particle Hamiltonian $\mathbf{h}^{0}$ reads

$$
\mathbf{h}^{0}=\hbar\left(\begin{array}{cc}
\omega_{X}(-i \nabla) & \Omega_{R} \\
\Omega_{R} & \omega_{C}(-i \nabla)
\end{array}\right)
$$

where $\omega_{C}(\mathbf{k})=\omega_{C}^{0} \sqrt{1+\mathbf{k}^{2} / k_{z}^{2}}$ is the cavity mode energy dispersion as a function of the in-plane wavevector $\mathbf{k}$ and $k_{z}$ is the quantized photon wavevector along the growth direction. $\Omega_{R}$ is the Rabi frequency of the exciton-cavity photon coupling. In the following, we will consider a flat exciton dispersion $\omega_{X}(\mathbf{k})=\omega_{X}$. In this framework of coupled harmonic oscillators, polaritons simply arise as the eigenstates of the linear Hamiltonian (2). $\omega_{L P(U P)}(\mathbf{k})$ denotes the dispersion of the lower (upper) polariton branch [Fig प(a)].

The term proportional to $F_{p}(\mathbf{x}, t)$ in Eq. (1I) represents an applied coherent laser pump spot, which drives the cavity and injects polaritons. In the following, we will consider the case of a monochromatic laser field of frequency $\omega_{p}$ and plane-wave profile with in-plane wave-vector $\mathbf{k}_{p}$. The in-plane wave-vector is linked to the incident direction by the simple relationship $k_{p}=$ $\sin \theta_{p} \omega_{p} / c, \theta_{p}$ being the pump incidence angle with respect to the growth direction. This means that an oblique pump incidence generates a polariton fluid with a nonzero flow velocity along the cavity plane. For $\hbar \omega_{p}=1400$ $\mathrm{meV}$, an in-plane wavevector $k_{p}=1(\mu \mathrm{m})^{-1}$ corresponds to a pump incidence angle of $8.1^{\circ}$.

The nonlinear interaction term in Eq. (11) is due to exciton-exciton collisional interactions and, as usual, is modelled by a repulsive $(g>0)$ contact potential. The anharmonic exciton-photon coupling has a negligible ef-

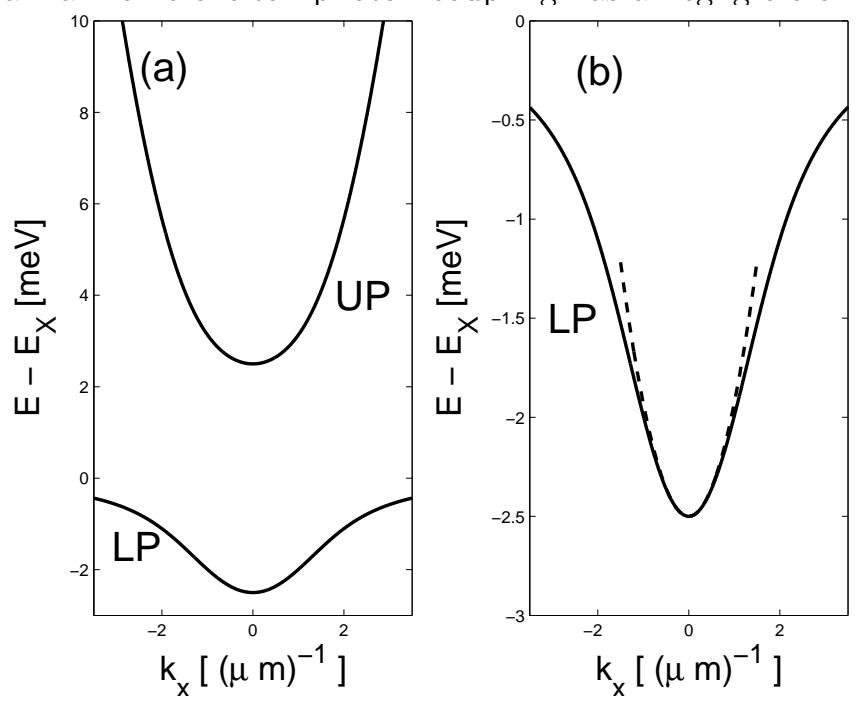

FIG. 1: (a): Linear dispersion of the Lower (LP) and Upper (UP) Polariton branches as a function of $k_{x}\left(k_{y}=0\right)$. Cavity parameters: $\hbar \omega_{X}=\hbar \omega_{C}=1.4 \mathrm{eV}, 2 \hbar \Omega_{R}=5 \mathrm{meV}$. (b) Zoom of the LP branch. The dashed line depicts the parabolic approximation around the bottom of the dispersion.

fect in the regime considered in the present study 31. and will be neglected for sake of clarity.

Finally, $V_{X, C}(\mathbf{x})$ are the single particle potentials acting on the exciton and photon fields respectively. These potentials break the translational symmetry along the cavity plane. The exciton potential $V_{X}(\mathbf{x})$ can be due to natural interface or alloy disorder in the semiconductor quantum wells due to unavoidable growth imperfections. The photonic potential $V_{C}(\mathbf{x})$ can be due to fluctuations of the cavity length or imperfections in the Bragg reflectors (photonic disorder 24]). More interestingly, $V_{C}(\mathbf{x})$ can be designed and created deliberately by means of lithographic techniques.

Within the mean-field approximation, the timeevolution of the mean fields $\psi_{X, C}(\mathbf{x})=\left\langle\hat{\Psi}_{X, C}(\mathbf{x})\right\rangle$ under the Hamiltonian (11) is given by:

$$
i \frac{d}{d t}\left(\begin{array}{c}
\psi_{X}(\mathbf{x}) \\
\psi_{C}(\mathbf{x})
\end{array}\right)=\left(\begin{array}{c}
0 \\
F_{p} e^{i\left(\mathbf{k}_{p} \mathbf{x}-\omega_{p} t\right)}
\end{array}\right)+\left[\mathbf{h}^{0}+\left(\begin{array}{cc}
V_{X}(\mathbf{x})+g\left|\psi_{X}(\mathbf{x})\right|^{2}-i \gamma_{X} & 0 \\
0 & V_{C}(\mathbf{x})-i \gamma_{C}
\end{array}\right)\right]\left(\begin{array}{c}
\psi_{X}(\mathbf{x}) \\
\psi_{C}(\mathbf{x})
\end{array}\right) .
$$



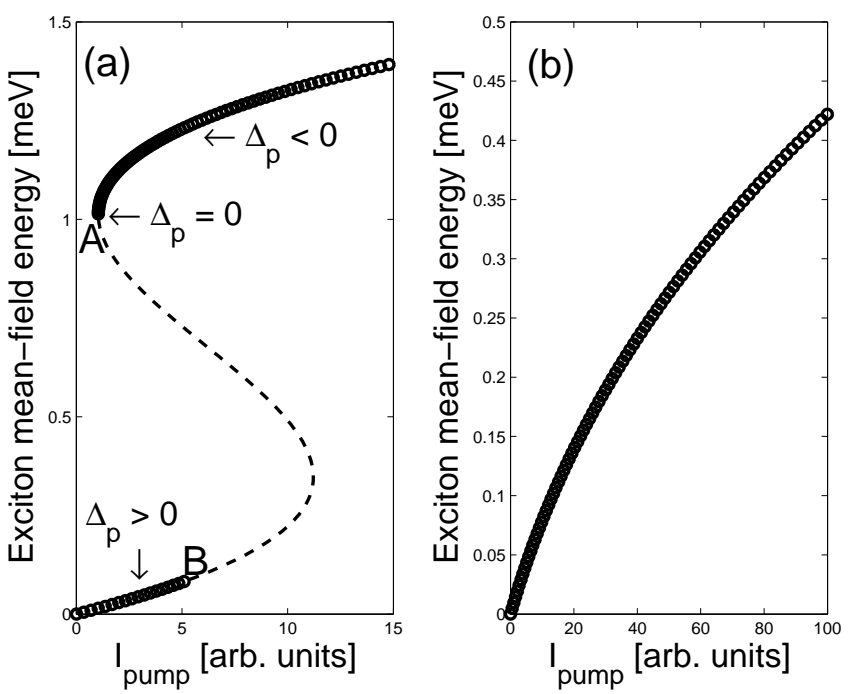

FIG. 2: Exciton mean-field energy $\hbar g\left|\Psi_{X}^{s s}\right|^{2}$ (meV) as a function of the incident pump intensity (arb. units). Cavity parameters: $\hbar \gamma_{C}=\hbar \gamma_{X}=0.1 \mathrm{meV}, \hbar \omega_{X}=\hbar \omega_{C}^{0}=1.4 \mathrm{eV}$, $2 \hbar \Omega_{R}=5 \mathrm{meV}$. (a) Bistability curve obtained with the excitation parameters: $k_{p}=0.314 \mu \mathrm{m}^{-1}$ (well in the parabolic region near the bottom of the LP dispersion), $\hbar \omega_{p}-\hbar \omega_{L P}\left(\mathbf{k}_{p}\right)=$ $0.47 \mathrm{meV}$. Circles depict the calculated stable points, while the dashed line represents the unstable branch. The threshold points $\mathrm{A}$ and $\mathrm{B}$ are, respectively, due to a single-mode (Kerr) or a multi-mode (parametric) instability. (b) Optical limiter curve obtained with the same $k_{p}$, but with $\hbar \omega_{p}-\hbar \omega_{L P}\left(\mathbf{k}_{p}\right)=-0.47 \mathrm{meV}$. In this case, all stationary solutions are stable.

cavity-polariton system. The quantities $\gamma_{X}$ and $\gamma_{C}$ represent the homogoneous broadening of the exciton and photon modes respectively. In the present work, we will be concerned with an excitation close to the bottom of the LP dispersion, i.e. the region most protected [5] from the exciton reservoir, which may be otherwise responsible for excitation-induced decoherence 32].

\section{STATIONARY SOLUTIONS IN THE HOMOGENEOUS CASE}

In the homogeneous case (i.e., $V_{X, C}(\mathbf{x})=0$ and translational invariance along the plane), we can look for spatially homogeneous stationary states of the system in which the field has the same plane-wave structure $\psi_{X, C}(\mathbf{x}, t)=\exp \left[i\left(\mathbf{k}_{p} \mathbf{x}-\omega_{p} t\right)\right] \psi_{X, C}^{s s}$ as the incident laser pump field. The mean-field equations

$$
\begin{array}{r}
\left(\omega_{X}\left(\mathbf{k}_{p}\right)-\omega_{p}-\frac{i}{2} \gamma_{X}+g\left|\psi_{X}^{s s}\right|^{2}\right) \psi_{X}^{s s}+\Omega_{R} \psi_{C}^{s s}=0 \\
\left(\omega_{C}\left(\mathbf{k}_{p}\right)-\omega_{p}-\frac{i}{2} \gamma_{C}\right) \psi_{C}^{s s}+\Omega_{R} \psi_{X}^{s s}=-F_{p}
\end{array}
$$

are the non-equilibrium analogous of the state equation, which in equilibrium systems links the chemical potential to the particle density. Importantly, we stress that while the oscillation frequency of the condensate wavefunction in an isolated gas is equal to the chemical potential $\mu$ and therefore it is fixed by the equation of state, in the present driven-dissipative system it is equal to the frequency $\omega_{p}$ of the driving laser and therefore it is an experimentally tunable parameter. Hence, the microcavity polariton system allows us to explore a regime, which is not accessible in systems close to thermal equilibrium, such as the ultracold trapped atoms.

\section{LINEARIZED BOGOLIUBOV-LIKE THEORY}

As usual in the theory of nonlinear systems, stability of the solutions of Eqs. (45) with respect to fluctuations has to be checked by linearizing Eq. (3) around the stationary state. Perturbations can be produced by classical fluctuations of the pump field, quantum noise of the exciton and photon fields as well as the presence of perturbing potentials $V_{C, X}(\mathbf{x})$, which have not been considered by the plane-wave solutions in Eqs. (45).

In the stability region, the linearized response of the system to a weak perturbation is analogous to the celebrated Bogoliubov theory of the weakly interacting Bose gas [2]. Let us define the slowly varying fields with respect to the pump frequency as

$$
\delta \phi_{i}(\mathbf{x}, t)=\delta \psi_{i}(\mathbf{x}, t) \exp \left(i \omega_{p} t\right)
$$

and let us consider the four-component displacement vector

$$
\delta \vec{\phi}(\mathbf{x}, t)=\left(\delta \phi_{X}(\mathbf{x}, t), \delta \phi_{C}(\mathbf{x}, t), \delta \phi_{X}^{*}(\mathbf{x}, t), \delta \phi_{C}^{*}(\mathbf{x}, t)\right)^{T} .
$$

The equation of motion for the four-component displacement vector reads

$$
i \frac{d}{d t} \delta \vec{\phi}=\mathcal{L} \cdot \delta \vec{\phi}+\vec{f}_{\text {pert }},
$$

where $\vec{f}_{\text {pert }}$ is the inhomogeneous source term produced by the perturbation. The expression for $\vec{f}_{\text {pert }}$ depends on which kind of perturbation is considered and will be given explicitly later for the case of a perturbation induced by the single particle potentials $V_{C, X}(\mathbf{x})$. The linear operator $\mathcal{L}$ is 
$\mathcal{L}=\left(\begin{array}{cc}\omega_{X}+2 g\left|\psi_{X}^{s s}\right|^{2}-\omega_{p}-\frac{i \gamma_{X}}{2} & \Omega_{R} \\ \Omega_{R} & \omega_{C}(-i \nabla)-\omega_{p}-\frac{i \gamma_{C}}{2} \\ -g \psi_{X}^{s s * 2} e^{-2 i \mathbf{k}_{p} \mathbf{x}} & 0 \\ 0 & 0\end{array}\right.$
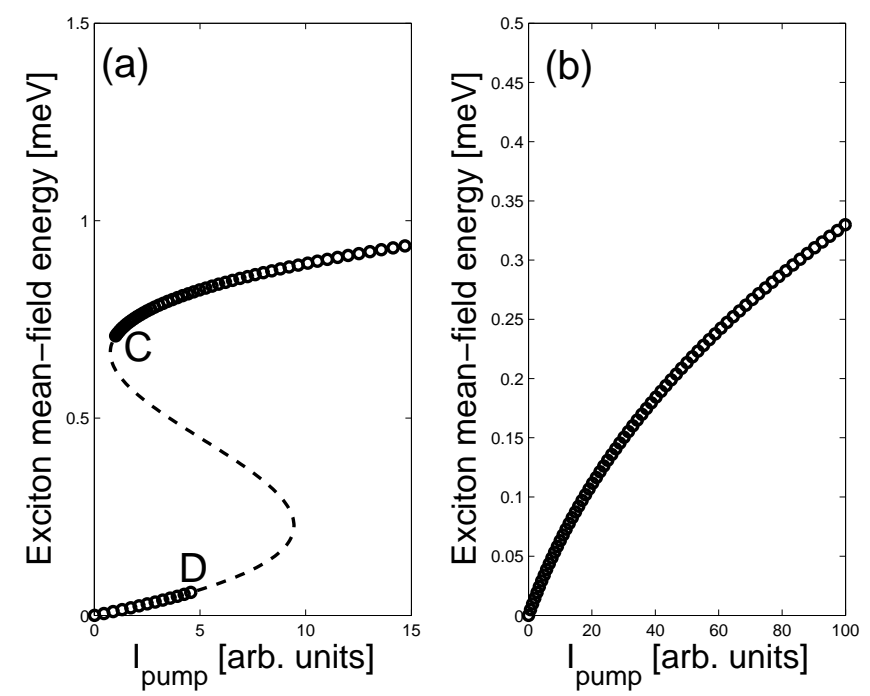

FIG. 3: Same plot as in Fig. 2 but with pump in-plane wavevector $k_{p}=1.5 \mu \mathrm{m}^{-1}$ (close to the inflection point of the LP dispersion). Pump frequency: (a) $\hbar \omega_{p}-\hbar \omega_{L P}\left(\mathbf{k}_{p}\right)=$ $0.47 \mathrm{meV}$, (b) $\hbar \omega_{p}-\hbar \omega_{L P}\left(\mathbf{k}_{p}\right)=-0.47 \mathrm{meV}$. In contrast to Fig. 2 here both threshold points $\mathrm{C}$ and $\mathrm{D}$ are due to parametric instabilities.

\section{A. Stability of the stationary solutions}

The stability of the solutions of Eqs. (4.5) can be determined by calculating the imaginary parts of the eigenvalues of the operator $\mathcal{L}$. If all the eigenvalues have negative imaginary parts (i.e., as it happens in the noninteracting case), then the solutions are stable. Otherwise, an instability occurs. If the polariton instability involves only the pump mode, we have the analogous of a Kerr instability. If the instability is due to pairs of modes (formation of the so-called signal-idler pairs), we have a parametric instability (in the field of quantum fluids, this kind of dynamical instabilities are generally known as modulational instabilities [33]). In Fig. 2 we have plotted the stationary solutions for the exciton mean-field energy $\hbar g\left|\psi_{X}^{s s}\right|^{2}$ (meV) as a function of the incident pump intensity (arb. units) for realistic microcavity parameters and with a small pump wave-vector $\left(k_{p}=0.314 \mu \mathrm{m}^{-1}\right)$, close to the bottom of the LP dispersion. In Fig. [2(a), we have considered the case of a pump frequency, which is blue-detuned with respect to the unperturbed lower

$$
\left.\begin{array}{cc}
g \psi_{X}^{s s} 2 e^{2 i \mathbf{k}_{p} \mathbf{x}} & 0 \\
0 & 0 \\
-\left(\omega_{X}+2 g\left|\psi_{X}^{s s}\right|^{2}\right)+\omega_{p}-\frac{i \gamma_{X}}{2} & -\Omega_{R} \\
-\Omega_{R} & -\omega_{C}(-i \nabla)+\omega_{p}-\frac{i \gamma_{C}}{2}
\end{array}\right) .
$$

polariton energy $\left(\omega_{p}>\omega_{L P}\left(\mathbf{k}_{p}\right)\right)$. In this case, there is a clear S-shaped bistability curve 34, 35, 36, 37]. The unstable branch, determined through the eigenvalues of the linear operator $\mathcal{L}$, have been depicted with a dashed line, while the stable points are represented by circles. The threshold points A and B are due to a Kerr and to parametric instability respectively. By comparison, in Fig. 2(b), we have shown the same quantity, but for a reddetuned laser frequency $\left(\omega_{p}<\omega_{L P}\left(\mathbf{k}_{p}\right)\right)$. In this case, the polariton system behaves as an optical limiter [37], the absorption is highly sublinear and all the points are stable. To give a more complete picture, we report in Fig. 3. the analogous calculations, but with a larger wavevector $\left(k_{p}=1.5 \mu \mathrm{m}^{-1}\right)$, close to the inflection point of the LP dispersion. It is apparent that, while the shape is analogous, the boundary between the stable and unstable branches is modified. In particular, the threshold points $\mathrm{C}$ and $\mathrm{D}$ are both due to parametric instabilities. Note that nice hysteresis loops due to polariton bistability have been recently experimentally demonstrated in the case $k_{p}=0$ [34] and for a pump wavevector close to the inflection point of the LP dispersion [36].

\section{B. Complex energy of the collective excitations}

The spectrum of the collective excitations (Bogoliubov modes in the quantum fluid terminology) can be obtained from the eigenvalues of the operator $\mathcal{L}$. As the system is translationally invariant along the plane (we are considering the homogeneous case $V_{X}=V_{C}=0$ ), the wavevector $\mathbf{k}$ is a good quantum number and the eigenvectors of $\mathcal{L}$ have a plane-wave form

$$
\delta \vec{\phi}_{j, \mathbf{k}}^{ \pm}(\mathbf{x})=\left(\begin{array}{c}
u_{j_{,} X, \mathbf{k}}^{ \pm} e^{i \mathbf{k x}} \\
u_{j, C, \mathbf{k}}^{ \pm} e^{i \mathbf{k x}} \\
v_{j, X, \mathbf{k}}^{ \pm} e^{i\left(\mathbf{k}-2 \mathbf{k}_{p}\right) \mathbf{x}} \\
v_{j, C, \mathbf{k}}^{ \pm} e^{i\left(\mathbf{k}-2 \mathbf{k}_{p}\right) \mathbf{x}}
\end{array}\right)
$$

satisfying the reduced eigenvalue equation

$$
\left(\left(\omega_{j}^{ \pm}(\mathbf{k})-\omega_{p}\right) \mathbf{1}-\tilde{\mathcal{L}}\left(\mathbf{k}, \mathbf{k}_{p}\right)\right) \cdot\left(\begin{array}{c}
u_{j, X, \mathbf{k}}^{ \pm} \\
u_{j, C, \mathbf{k}}^{ \pm} \\
v_{j, X, \mathbf{k}}^{ \pm} \\
v_{j, C, \mathbf{k}}^{ \pm}
\end{array}\right)=0
$$




$$
\tilde{\mathcal{L}}\left(\mathbf{k}, \mathbf{k}_{p}\right)=\left(\begin{array}{cccc}
\omega_{X}+2 g\left|\psi_{X}^{s s}\right|^{2}-\frac{i \gamma_{X}}{2} & \Omega_{R} & g \psi_{X}^{s 2} & 0 \\
\Omega_{R} & \omega_{C}(\mathbf{k})-\frac{i \gamma_{C}}{2} & 0 & 0 \\
-g \psi_{X}^{s s * 2} & 0 & 2 \omega_{p}-\left(\omega_{X}+2 g\left|\psi_{X}^{s s}\right|^{2}\right)-\frac{i \gamma_{X}}{2} & -\Omega_{R} \\
0 & 0 & -\Omega_{R} & 2 \omega_{p}-\omega_{C}\left(2 \mathbf{k}_{p}-\mathbf{k}\right)-\frac{i \gamma_{C}}{2}
\end{array}\right)
$$

For each $\mathbf{k}$, the spectrum is composed by four branches. For each polariton branch $j \in\{L P, U P\}$, two \pm branches exist, which are related by the symmetry

$$
\omega_{j}^{-}(\mathbf{k})=2 \omega_{p}-\omega_{j}^{+}\left(2 \mathbf{k}_{p}-\mathbf{k}\right) .
$$

Now, we wish to point out and list clearly the relevant properties and symmetries in this problem. These properties will be later discussed in detail through a set of comprehensive examples and elucidations.

(i) The collective excitations are characterized by the pump-induced coherent coupling between a generic mode with wavevector $\mathbf{k}$ and the "idler" wavevector $2 \mathbf{k}_{p}-\mathbf{k}$. This corresponds to the elementary process $\left\{\mathbf{k}_{p}, \mathbf{k}_{p}\right\} \rightarrow$ $\left\{\mathbf{k}, 2 \mathbf{k}_{p}-\mathbf{k}\right\}$, i.e. the conversion of two pump excitations into a signal-idler pair (to use the quantum optics terminology of parametric oscillators) with the same total momentum.

(ii) The "idler" branch $\omega_{L P}^{-}(\mathbf{k})$ is the "image" of the ordinary branch $\omega_{L P}^{+}(\mathbf{k})$ under the simultaneous transformations [38] $\mathbf{k} \rightarrow 2 \mathbf{k}_{p}-\mathbf{k}$ and $\omega \rightarrow 2 \omega_{p}-\omega$. The same relationship holds for the UP branches $\omega_{U P}^{ \pm}(\mathbf{k})$.

(iii) The matrix $\tilde{\mathcal{L}}\left(\mathbf{k}, \mathbf{k}_{p}\right)$ is characterized by an antihermitian coupling between $\mathbf{k}$ and $2 \mathbf{k}_{p}-\mathbf{k}$. This feature is typical of parametric wave-mixing coupling (in the quantum optics language) or Bogoliubov theory (using the quantum fluid literature terminology).

(iv) The four branches of eigenvalues $\omega_{U P, L P}^{ \pm}(\mathbf{k})$ are complex. As the real parts, the imaginary parts of the eigenvalues depend both on $\mathbf{k}$ and on the pump parameters. In the stability region, all imaginary parts are negative.

(v) In case of resonant excitation of the lower branch, provided that the interaction energy $g\left|\psi_{X}^{s s}\right|^{2}$ is much smaller than the polaritonic splitting $\omega_{U P}-\omega_{L P}$, there is no significant mixing between the LP and UP branches. Hence, a simplified approach consists in neglecting the contribution of the upper branch. With this approximation, the branches $\omega_{L P}^{ \pm}(\mathbf{k})$ are the eigenvalues of the simplified matrix

$$
\tilde{\mathcal{L}}_{L P}\left(\mathbf{k}, \mathbf{k}_{p}\right)=\left(\begin{array}{cc}
\omega_{L P}(\mathbf{k})+2 g_{L P}\left|\psi_{L P}^{s s}\right|^{2}-\frac{i \gamma_{L P}(\mathbf{k})}{2} & g_{L P} \psi_{L P}^{s s} 2 \\
-g_{L P} \psi_{L P}^{s s * 2} & 2 \omega_{p}-\left(\omega_{L P}\left(2 \mathbf{k}_{p}-\mathbf{k}\right)+2 g_{L P}\left|\psi_{L P}^{s s}\right|^{2}\right)-\frac{i \gamma_{L P}(\mathbf{k})}{2}
\end{array}\right)
$$

where the stationary lower polariton field is written as a linear superposition of the exciton and cavity photon fields, namely

$$
\psi_{L P}^{s s}=X_{L P}\left(\mathbf{k}_{p}\right) \psi_{X}^{s s}+C_{L P}\left(\mathbf{k}_{p}\right) \psi_{C}^{s s},
$$

being $\left|X_{L P}\left(\mathbf{k}_{p}\right)\right|^{2}$ and $\left|C_{L P}\left(\mathbf{k}_{p}\right)\right|^{2}$ the exciton and photon fractions of the lower polariton mode with the pump wavevector. The effective interaction strength

$$
g_{L P}=g\left|X_{L P}\left(\mathbf{k}_{p}\right)\right|^{2} X_{L P}(\mathbf{k}) X_{L P}\left(2 \mathbf{k}_{p}-\mathbf{k}\right)
$$

takes into account for the exciton fraction of the involved lower polariton modes (pump, signal and idler). The polariton linewidth is given by $\gamma_{L P}(\mathbf{k})=\left|X_{L P}(\mathbf{k})\right|^{2} \gamma_{X}+$ $\left|C_{L P}(\mathbf{k})\right|^{2} \gamma_{C}$.

(vi) When the diagonal elements of $\tilde{\mathcal{L}}_{L P}\left(\mathbf{k}, \mathbf{k}_{p}\right)$ are equal, it is easy to verify that $\Re\left[\omega_{L P}^{+}(\mathbf{k})\right]=\Re\left[\omega_{L P}^{-}(\mathbf{k})\right]$, while $\Im\left[\omega_{L P}^{+}(\mathbf{k})\right] \neq \Im\left[\omega_{L P}^{-}(\mathbf{k})\right]$. This means that the parametric coupling produces a splitting of the imaginary parts of the two LP branches, while the real parts are the same. If the difference between the diagonal elements of $\tilde{\mathcal{L}}_{L P}\left(\mathbf{k}, \mathbf{k}_{p}\right)$ is small compared to the coupling $g_{L P}\left|\psi_{L P}^{s s}\right|^{2}$, then the same property holds. In other words, the dispersions of the two branches $\omega_{L P}^{+}(\mathbf{k})$ and $\omega_{L P}^{-}(\mathbf{k})$ stick together, while their imaginary parts are split. One branch is narrowed with respect to the linear regime, while the other is overdamped. Analogous properties occur for the exact eigenvalues of the $4 \times 4$ matrix $\tilde{\mathcal{L}}\left(\mathbf{k}, \mathbf{k}_{p}\right)$, which are reported in all the figures of this paper.

\section{Excitation near the inflection point of the LP dispersion}

In the following, we will show the exact eigenvalues (obtained by numerical calculations) of the matrix $\tilde{\mathcal{L}}\left(\mathbf{k}, \mathbf{k}_{p}\right)$ in Eq. (12) as a function of the excitation parameters (pump frequency, wavevector and intensity). We will focus on the subtle interplay between the dra- 

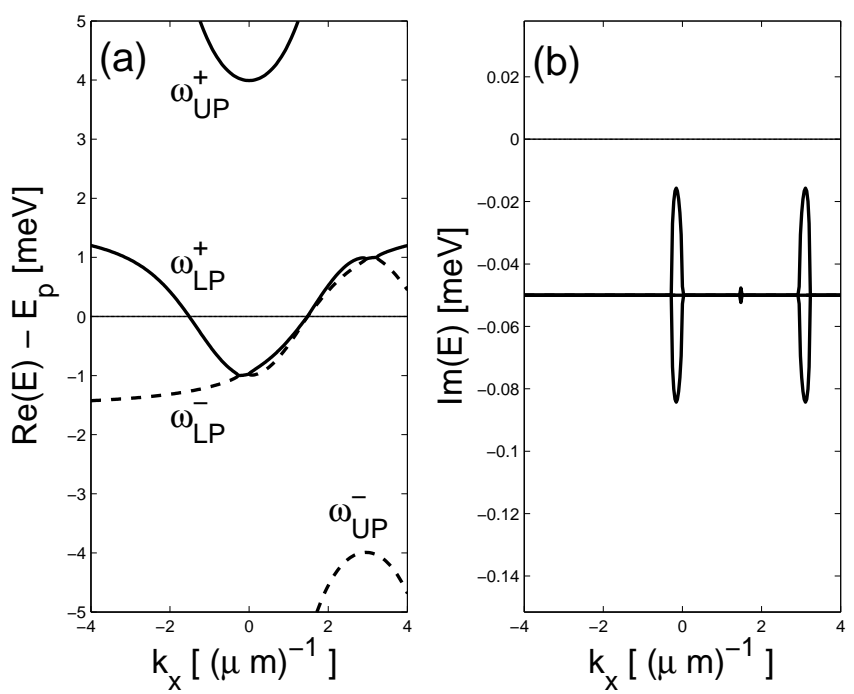

FIG. 4: (a) Exact energy dispersions $\Re\left[\hbar \omega_{L P, U P}^{ \pm}\right]$of the four polariton Bogoliubov branches measured with respect to the pump photon energy $\hbar \omega_{p}(\mathrm{meV})$. (b) Corresponding imaginary parts $(\mathrm{meV})$. Note that negative imaginary parts imply stability. Excitation parameters: $k_{p}=1.5 \mu \mathrm{m}^{-1}$ (along the $\mathrm{x}$-axis), $\hbar \omega_{p}-\hbar \omega_{L P}\left(\mathbf{k}_{p}\right)=0.107 \mathrm{meV}, \hbar g\left|\Psi_{X}^{s s}\right|^{2}=0.05 \mathrm{meV}$. Cavity parameters as in the previous figures.
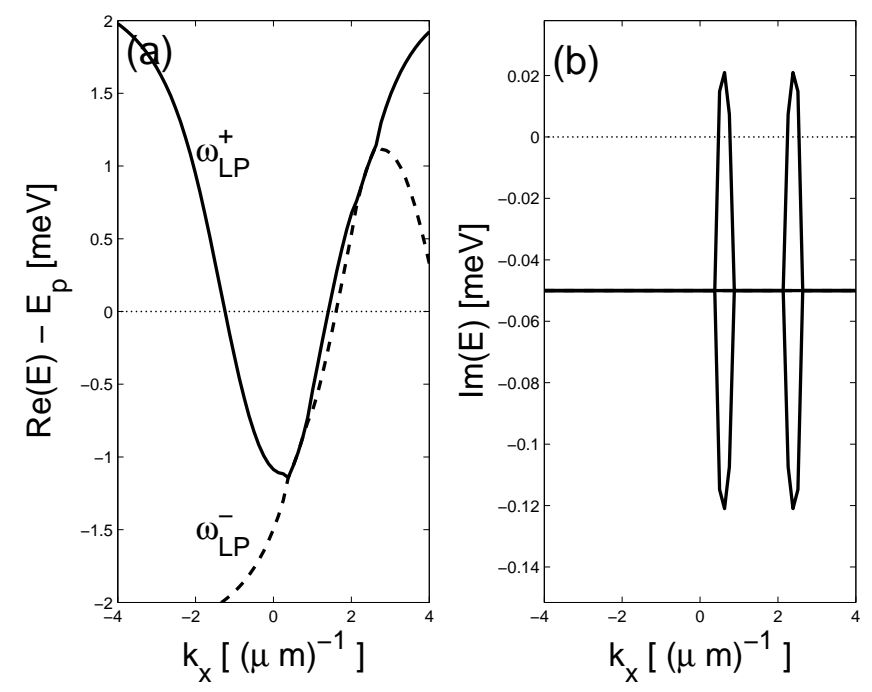

FIG. 5: (a) Exact energy dispersions $\Re\left[\hbar \omega_{L P}^{ \pm}\right]$of the lower polariton Bogoliubov branches measured with respect to the pump photon energy $\hbar \omega_{p}(\mathrm{meV})$. (b) Corresponding imaginary parts $(\mathrm{meV})$. Excitation parameters: $k_{p}=1.5 \mu \mathrm{m}^{-1}$ (along the x-axis), $\hbar \omega_{p}-\hbar \omega_{L P}\left(\mathbf{k}_{p}\right)=0.47 \mathrm{meV}, \hbar g\left|\Psi_{X}^{s s}\right|^{2}=$ $0.699 \mathrm{meV}$. Note that here the stationary solution is unstable, because there are modes with positive imaginary parts. This unstable point is close to the point C in Fig. 31 a).

matic modification of the energy dispersions (depending on the real part of the eigenvalues) and the onset of
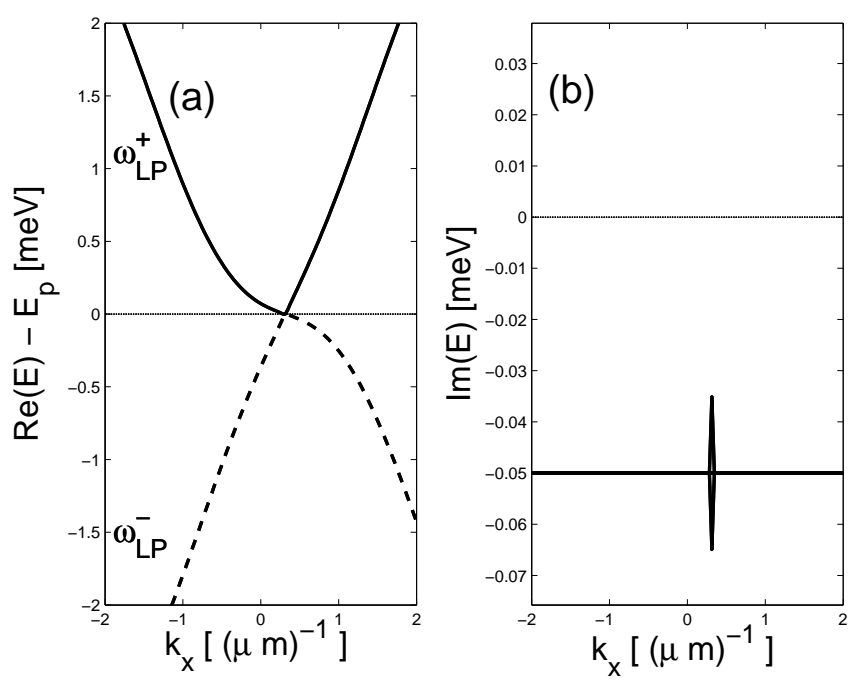

FIG. 6: (a) Exact energy dispersions $\Re\left[\hbar \omega_{L P}^{ \pm}\right]$of the lower polariton Bogoliubov branches measured with respect to the pump photon energy $\hbar \omega_{p}(\mathrm{meV})$. (b) Corresponding imaginary parts $(\mathrm{meV})$. Excitation parameters: $k_{p}=0.314 \mu \mathrm{m}^{-1}$ (along the x-axis), $\hbar \omega_{p}-\hbar \omega_{L P}\left(\mathbf{k}_{p}\right)=0.47 \mathrm{meV}, \hbar g\left|\Psi_{X}^{s s}\right|^{2}=$ $1.02 \mathrm{meV}$. Note that this case is the precursor of a Kerr instability, because the imaginary parts are modified at the pumped mode only. This stable point is close to the inversion point $\mathrm{A}$ in Fig. 2(a).

the parametric instabilities (depending on the imaginary part).

As a first example, we consider the case of nearlyresonant excitation close to the inflection point of the LP dispersion (see Fig. [4). The pump frequency has been taken slightly blue-detuned with respect to the polariton energy in the linear regime. In Fig. 廿4 a) the exact dispersions of the four polariton Bogoliubov branches is shown. The upper polariton branches are energetically far away and play a negligible role, while the relevant physics concerns the lower polariton branches $\omega_{L P}^{ \pm}$only. The corresponding imaginary parts are shown in $4(b)$. It is apparent that there is a dramatic modification of the imaginary part around the wave-vectors $k_{x}=0$ and $k_{x}=2 k_{p}$. Although the stationary solutions are here stable (negative imaginary parts), we can see that we are close to a parametric instability. In fact, there is one branch, whose imaginary part is not far from zero. Note that the imaginary parts are split at $\mathbf{k}_{p}$ as well, even if in a much weaker way. This is a precursor of a Kerr (or single-mode) instability.

In Fig. 5 we give another example, with the same excitation parameters as in Fig. 3(a) and with an exciton mean-field energy $\hbar g\left|\Psi_{X}^{s s}\right|^{2}=0.699 \mathrm{meV}$. In this case, we have an unstable solution, because, as shown in Fig there are modes with positive imaginary parts. On the bistable curve of Fig. 3(a), we are here just beyond $C$. The instability being of parametric (or multi-mode) type, 

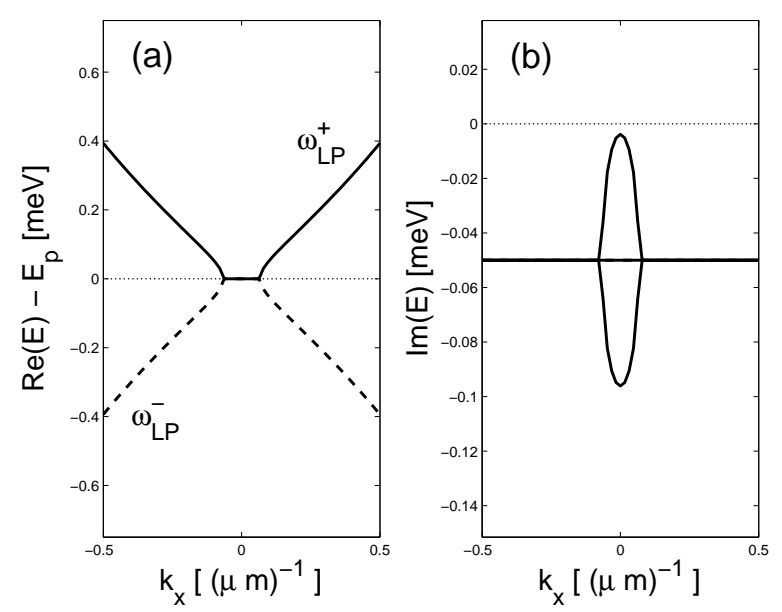

FIG. 7: (a) Exact energy dispersions $\Re\left[\hbar \omega_{L P}^{ \pm}\right]$of the lower polariton Bogoliubov branches measured with respect to the pump photon energy $\hbar \omega_{p}(\mathrm{meV})$. (b) Corresponding imaginary parts $(\mathrm{meV})$. Excitation parameters: $k_{p}=0, \hbar \omega_{p}-$ $\hbar \omega_{L P}\left(\mathbf{k}_{p}\right)=0.532 \mathrm{meV}, \hbar g\left|\Psi_{X}^{s s}\right|^{2}=1.2 \mathrm{meV}$.

note that the point $C$ is close but does not coincide with the inversion point of the bistable curve. In Fig. 5 (a), we can see that the branches $\omega_{L P}^{ \pm}$stick together in the wavevector region where the parametric instability takes place.

\section{Excitation near the bottom of the LP dispersion}

Here, we consider the case of a smaller pump excitation wavevector and energy, such as to excite the LP branch close to the bottom of its dispersion. In this region, as shown by Fig. T(b), the dispersion of the unperturbed lower polariton branch is parabolic. In order to stress the non-trivial effects here predicted, we start by showing a spectacular case, depicted in Fig [6] The excitation parameters correspond to Fig. [2] (a) with the exciton meanfield energy $\hbar g\left|\Psi_{X}^{s s}\right|^{2}=1.02 \mathrm{meV}$, i.e., a point close to the threshold point $\mathrm{A}$ for the Kerr instability. The dispersions of the branches $\omega_{L P}^{ \pm}$in Fig 6 (a) have a corner at the pump wavevector. This dispersion is reminiscent of the celebrated Bogoliubov linear dispersion in superfluid helium and in the atomic condensates. If we look at Fig 6 (b), we realize that the modification of the imaginary part is peaked around the pump wavevector itself. Using the quantum optics language, this is the precursor of a Kerr instability, because it involves the pumped mode only.

In Fig. 7 we give another example, which has no analog in equilibrium systems. Here, we have a bluedetuned pump at normal incidence, namely $k_{p}=0$, $\hbar \omega_{p}-\hbar \omega_{L P}\left(\mathbf{k}_{p}\right)=0.53 \mathrm{meV}$ and $\hbar g\left|\Psi_{X}^{s s}\right|^{2}=1.2 \mathrm{meV}$. In Fig. 7(a), we can clearly see that the dispersion of the polariton collective excitations is flat around the pump
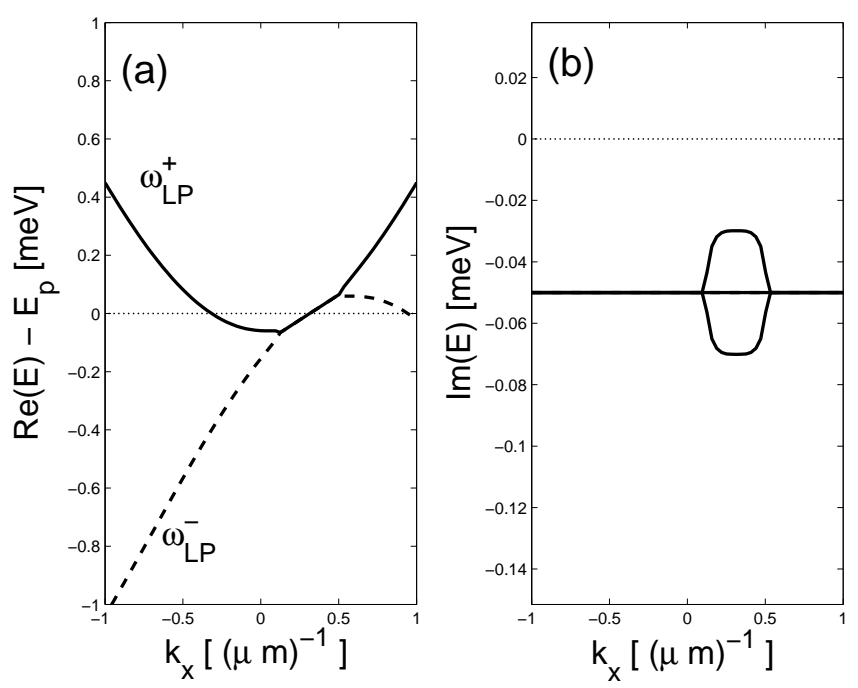

FIG. 8: (a) Exact energy dispersions $\Re\left[\hbar \omega_{L P}^{ \pm}\right]$of the lower polariton Bogoliubov branches measured with respect to the pump photon energy $\hbar \omega_{p}(\mathrm{meV})$. (b) Corresponding imaginary parts $(\mathrm{meV})$. Excitation parameters: $k_{p}=0.314 \mu \mathrm{m}^{-1}$ (along the x-axis), $\hbar \omega_{p}-\hbar \omega_{L P}\left(\mathbf{k}_{p}\right)=0.04 \mathrm{meV}, \hbar g\left|\Psi_{X}^{s s}\right|^{2}=$ $0.04 \mathrm{meV}$.
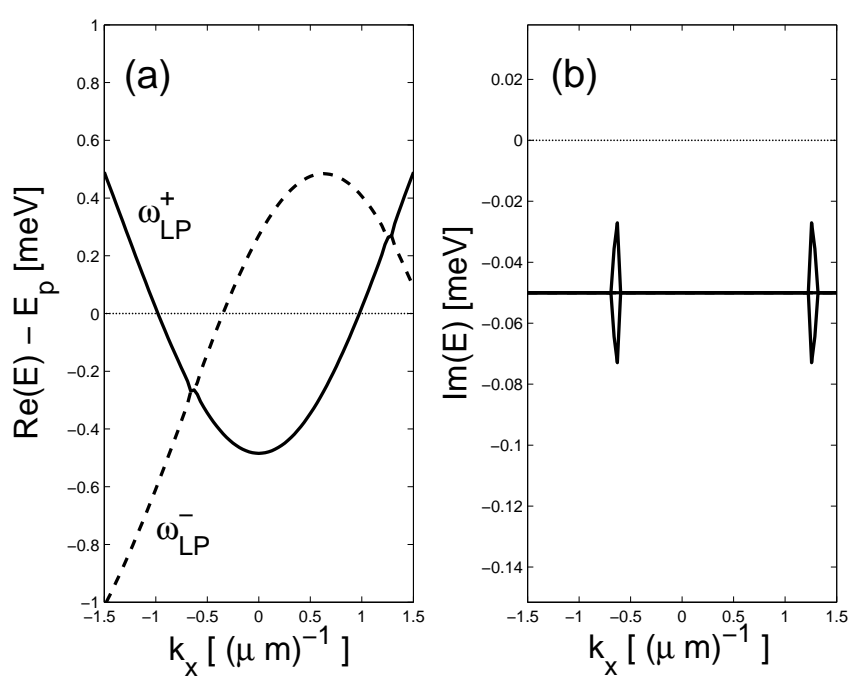

FIG. 9: (a) Exact energy dispersions $\Re\left[\hbar \omega_{L P}^{ \pm}\right]$of the lower polariton Bogoliubov branches measured with respect to the pump photon energy $\hbar \omega_{p}(\mathrm{meV})$. (b) Corresponding imaginary parts $(\mathrm{meV})$. Excitation parameters: $k_{p}=0.314 \mu \mathrm{m}^{-1}$ (along the x-axis), $\hbar \omega_{p}-\hbar \omega_{L P}\left(\mathbf{k}_{p}\right)=0.47 \mathrm{meV}, \hbar g\left|\Psi_{X}^{s s}\right|^{2}=$ $0.04 \mathrm{meV}$.

wave-vector. For the parameters of Fig. [7(b), the imaginary parts are all negative, which implies stability, but, as in Fig G (b), we are not far from the onset of a Kerr instability. Note that in Fig. 8 we have an analogous situation, but with a finite pump wavevector. As shown by Fig. 8 (a), the branches $\omega_{L P}^{ \pm}$stick together around the pump wavevector, with a dispersion exactly linear. 
In Fig. 9(a), we show the dispersions for a stable point, which is close to the threshold point B in Fig. [2 (a). Note that here the exciton mean-field energy is considerably smaller than the pump detuning. Hence, the branch sticking occurs in a limited portion of momentum space, where the imaginary parts are affected, as shown in Fig. 9(b).

Finally, in Fig. 10(a), we give another different example, with a full gap between the branch $\omega_{L P}^{+}$and the branch $\omega_{L P}^{-}$. In Fig. 10(b), we can see that the imaginary parts of the eigenvalues are unchanged with respect to the linear regime. Indeed, for these excitation parameters (see the caption of Fig. 10], the microcavity has an optical limiter behavior similar to the one in Fig. 2(b) and no instability occurs if the pump intensity is further increased.

\section{Simplified analytical model for excitation close to the bottom of the LP dispersion}

Now, after having shown a few examples of the rich spectra of non-equilibrium collective excitations and the variety of interaction-induced polariton instabilities, we present here a simple approximated approach, which allows us to grasp effectively the physics contained by the eigenvalues of the matrix in Eq. (12). In particular, we consider the case of negligible mixing with the UP branches, which allows us to focus our analysis on the simpler $2 \times 2$ matrix in Eq. (14) instead of the $4 \times 4$ matrix in Eq. (12). Moreover, we consider a pump excitation close to the bottom of the LP dispersion, where the dispersion is approximately parabolic (see Fig. 廿(b)), i.e.,

$$
\omega_{L P}(\mathbf{k}) \simeq \omega_{L P}(0)+\frac{\hbar \mathbf{k}^{2}}{2 m_{L P}}
$$

where $m_{L P}$ is the effective mass of the LP dispersion. Under these assumptions, the spectrum of the LP Bogoliubov excitations can be approximated by the simple expression

$$
\omega_{L P}^{ \pm} \simeq \omega_{p}+\delta \mathbf{k} \cdot \mathbf{v}_{p}-\frac{i \gamma_{L P}}{2} \pm \sqrt{\left(2 g_{L P}\left|\psi_{L P}^{s s}\right|^{2}+\eta_{\delta \mathbf{k}}-\Delta_{p}\right)\left(\eta_{\delta \mathbf{k}}-\Delta_{p}\right)}
$$

where $\delta \mathbf{k}=\mathbf{k}-\mathbf{k}_{p}$

$$
\eta_{\delta \mathbf{k}}=\frac{\hbar \delta \mathbf{k}^{2}}{2 m_{L P}}
$$

the pump mode flow velocity is $\mathbf{v}_{p}=\hbar \mathbf{k}_{p} / m_{L P}$ and the interaction-renormalized pump detuning

$$
\Delta_{p}=\omega_{p}-\omega_{L P}\left(\mathbf{k}_{p}\right)-g_{L P}\left|\psi_{L P}^{s s}\right|^{2} .
$$

In the case of resonant exciton-photon coupling (i.e. $\left.\omega_{C}(0)=\omega_{X}\right)$ and small wavevectors, the excitonic fraction of the lower polariton mode is approximately 0.5. Under these assumptions, we have $\left|\psi_{L P}^{s s}\right|^{2} \approx$ $2\left|\psi_{X}^{s s}\right|^{2}$ and $g_{L P} \approx g / 4$ [see Eqs. (1516)]. Therefore, $g_{L P}\left|\psi_{L P}^{s s}\right|^{2} \approx 0.5 g\left|\psi_{X}^{s s}\right|^{2}$, i.e. the mean-field interaction energy "felt" by the lower polariton is half of the mean-field energy for the exciton field. Note that there are three different cases, according to the value of $\Delta_{p}$ defined in Eq. (20).

(i) $\Delta_{p}=0$. In this resonant situation, the \pm branches touch at $\mathbf{k}=\mathbf{k}_{p}$. The effect of the finite flow velocity $\mathbf{v}_{p}$ is to tilt the standard Bogoliubov dispersion [2] via the term $\delta \mathbf{k} \cdot \mathbf{v}_{p}$. While in the non-interacting case the dispersion is parabolic, in the presence of interactions [Fig 6(a)] its slope has a discontinuity at $\mathbf{k}=\mathbf{k}_{p}$. On each side of the corner, the + branch starts linearly with group velocities respectively given by $v_{g}^{r, l}=c_{s} \pm v_{p}, c_{s}$
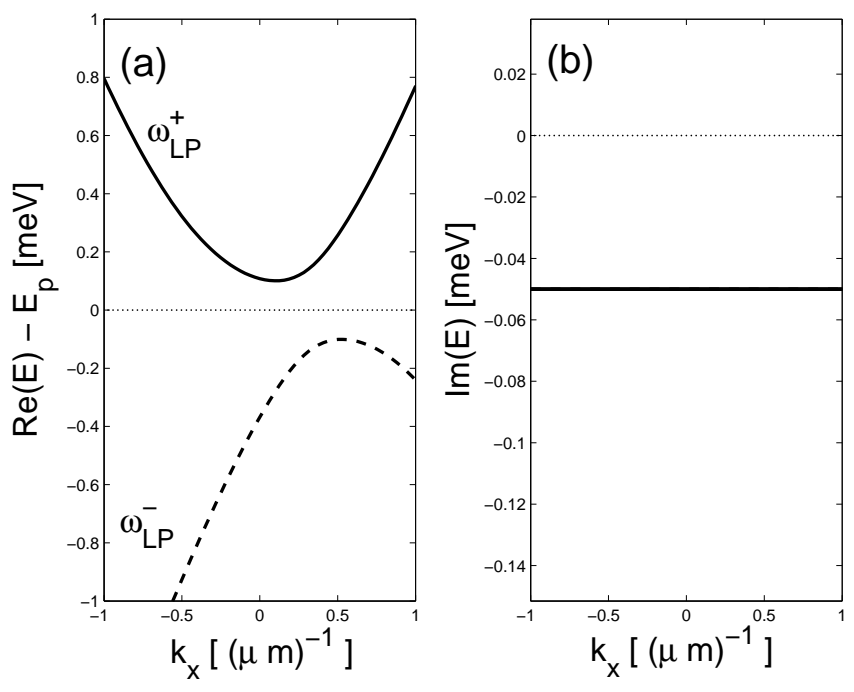

FIG. 10: (a) Exact energy dispersions $\Re\left[\hbar \omega_{L P}^{ \pm}\right]$of the lower polariton Bogoliubov branches measured with respect to the pump photon energy $\hbar \omega_{p}(\mathrm{meV})$. (b) Corresponding imaginary parts $(\mathrm{meV})$. Excitation parameters: $k_{p}=0.314 \mu \mathrm{m}^{-1}$ (along the x-axis), $\hbar \omega_{p}-\hbar \omega_{L P}\left(\mathbf{k}_{p}\right)=0.25 \mathrm{meV}, \hbar g\left|\Psi_{X}^{s s}\right|^{2}=$ $0.6 \mathrm{meV}$.

being the usual sound velocity of the interacting Bose gas 


$$
c_{s}=\sqrt{\hbar g_{L P}\left|\Psi_{L P}^{s s}\right|^{2} / m_{L P}} .
$$

On the hysteresis curve of Fig 2(a), the condition $\Delta_{p}=0$ corresponds to the inversion point $\mathrm{A}$.

(ii) $\Delta_{p}>0$. In this case, the argument of the square root in (18) is negative for the wavevectors $\mathbf{k}$ such that $\Delta_{p}>\eta_{\delta \mathbf{k}}>\Delta_{p}-2 g\left|\Psi_{X}^{s s}\right|^{2}$. In this region, the \pm branches stick together [31] (i.e. $\Re\left[\omega_{L P}^{+}\right]=\Re\left[\omega_{L P}^{-}\right]$) and have an exactly linear dispersion of slope $\mathbf{v}_{p}$ as in Fig. 8(a) and in Fig. 17(a). The imaginary parts are instead split, with one branch being narrowed and the other broadened 31, 38]. Increasing further the pump density, the multi-mode parametric instability [38] sets in, corresponding to the point B in Fig. 2(a).

(iii) $\Delta_{p}<0$. In this case, as it is shown in Fig 10 the branches no longer touch each other at $\mathbf{k}_{p}$ and a full gap between them opens up for sufficiently large values of $\left|\Delta_{p}\right|$. In Fig. 2(a), the region $\Delta_{p}<0$ is indicated.

\section{RESPONSE TO A STATIC POTENTIAL: RESONANT RAYLEIGH SCATTERING}

The dispersion of the polariton elementary excitations is the starting point for a study of the microcavity re- sponse to a perturbation. In particular, we shall consider here a moderate static disorder as described by the potential $V_{C, X}(\mathbf{x})$. In this case the perturbation source term for the equations of the linearized theory (see Eq. 8) is the time-independent quantity

$$
\vec{f}_{d}(\mathbf{x})=\left(\begin{array}{c}
V_{X}(\mathbf{x}) \phi_{X}^{s s} \\
V_{C}(\mathbf{x}) \phi_{C}^{s s} \\
-V_{X}(\mathbf{x}) \phi_{X}^{s s *} \\
-V_{C}(\mathbf{x}) \phi_{C}^{s s *}
\end{array}\right)
$$

The induced perturbation of the exciton and photon fields is given by the expression

$$
\delta \vec{\phi}_{d}(\mathbf{x})=-\mathcal{L}^{-1} \cdot \vec{f}_{d}(\mathbf{x})
$$

We remind you that, as shown by Eq. (9), $\mathcal{L}$ is an operator depending on the two-dimensional spatial gradient $\nabla$. It is convenient to perform a spatial Fourier transform, which leads to the algebraic result

$$
\left(\begin{array}{c}
\delta \tilde{\phi}_{X}(\mathbf{k}) \\
\delta \tilde{\phi}_{C}(\mathbf{k}) \\
\delta \tilde{\phi}_{X}^{*}\left(2 \mathbf{k}_{p}-\mathbf{k}\right) \\
\delta \tilde{\phi}_{C}^{*}\left(2 \mathbf{k}_{p}-\mathbf{k}\right)
\end{array}\right)=-\left(\tilde{\mathcal{L}}\left(\mathbf{k}, \mathbf{k}_{p}\right)-\hbar \omega_{p}\right)^{-1} \cdot\left(\begin{array}{c}
\tilde{V}_{X}(\mathbf{k}) \phi_{X}^{s s} \\
\tilde{V}_{C}(\mathbf{k}) \phi_{C}^{s s} \\
-\tilde{V}_{X}\left(\mathbf{k}-2 \mathbf{k}_{p}\right) \phi_{X}^{s s *} \\
-\tilde{V}_{X}\left(\mathbf{k}-2 \mathbf{k}_{p}\right) \phi_{C}^{s s *}
\end{array}\right)
$$

where the eigenvalues of the matrix $\tilde{\mathcal{L}}\left(\mathbf{k}, \mathbf{k}_{p}\right)$, defined in Eq. (12), are the 4 branches of polariton Bogoliubov excitations. The perturbation potentials $V_{C, X}(\mathbf{x})$ break the planar translational symmetry of the microcavity system, thus exciting polariton modes with in-plane wavevectors different from the pump wavevector $\mathbf{k}_{p}$. However, being $V_{C, X}(\mathbf{x})$ static, the resonant excitation concerns only Bogoliubov modes whose frequency is equal to $\omega_{p}$ (within the polariton homogeneous linewidth). The observable quantity is

$$
I_{R R S}(\mathbf{k}) \propto\left|\delta \tilde{\phi}_{C}(\mathbf{k})\right|^{2},
$$

i.e., the perturbation-induced intensity of the photonic field, which is proportional to the far-field images of the resonant Rayleigh scattering signal [14, 24, 29].

\section{A. Weak excitation regime and elastic RRS ring}

In the following, we will show a few applications of Eq. (24), using the perturbation potentials depicted in Fig. [11. Here, we have considered a single photonic defect (depth $1 \mathrm{meV}$, width $1.5 \mu \mathrm{m}$ ) for the in-plane photonic potential $V_{C}(\mathbf{x})$ [see Fig [11(a)] and a disordered excitonic potential [see Fig 111(b)]. We point out that these potentials are just an example and that Eq. (24) can be readily applied for an arbitrary set of perturbation potentials (see, e.g., the cover of this special volume). In the numerical applications here reported, we have considered a $256 \times 256$ spatial grid and a squared box $(400 \mu \mathrm{m} \times$ $400 \mu \mathrm{m}$ ), with the photonic dot at the center of the box.

In Fig. 12 we show the results for the weak excitation regime, where the many-body effects produced by polariton-polariton interactions are negligible. In this linear regime, we have the conventional unperturbed dispersions of the lower and upper polariton branches. In Fig. 12(a), we have considered the case of resonant exci- 

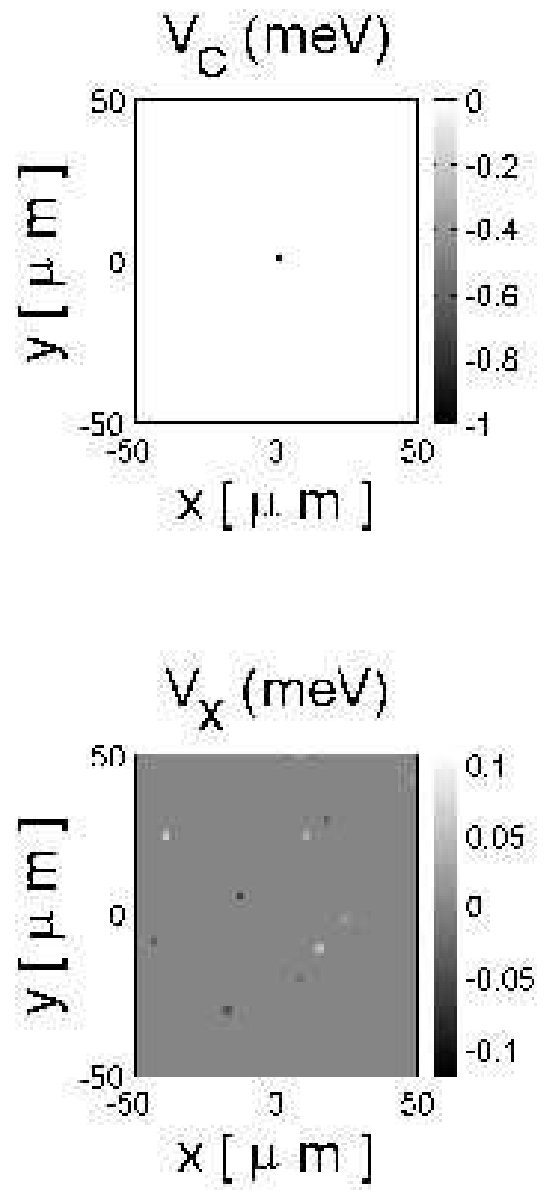

FIG. 11: Single particle potentials considered in the numerical calculations. Note that this is a zoom around the origin of the $400 \mu \mathrm{m} \times 400 \mu \mathrm{m}$ box (with a $256 \times 256$ grid) used for the numerical calculations. Top panel: Photonic potential $V_{C}(\mathbf{x})$ $(\mathrm{meV})$ (it can model an artificial or natural point defect at the origin $x=y=0$ ). Bottom panel: Excitonic potential $V_{X}(\mathbf{x})$ (disordered spatial fluctuations of the exciton energy). The gray color scale is different with respect to the top panel.

tation close to the bottom of the LP branch, where the dispersion is parabolic. In Fig. 12 (b), the intensity of the resonant Rayleigh scattering is shown, displaying the well known elastic ring. In fact, in the linear regime, the solutions of the equations $\omega_{L P}(\mathbf{k})=\omega_{p}$ are $\mathbf{k}$-points on a circle, because the unperturbed polariton dispersion depends on $|\mathbf{k}|$ only. The speckles on top of the elastic ring are due to the random nature of the excitonic potential. The width of the ring is due to the finite homogeneous broadening of the polariton modes. Note that, in order to excite the elastic ring corresponding to the wavevector $\mathbf{k}_{p}$, the Fourier component $\tilde{V}_{C, X}\left(\mathbf{k}_{p}\right)$ of the static potentials need to be finite. This condition is easily fulfilled by a typical excitonic disordered potential or by a photonic defect whose width is of the order of $1 \mu \mathrm{m}$.

In Fig. 13] we show the corresponding spatial pattern.
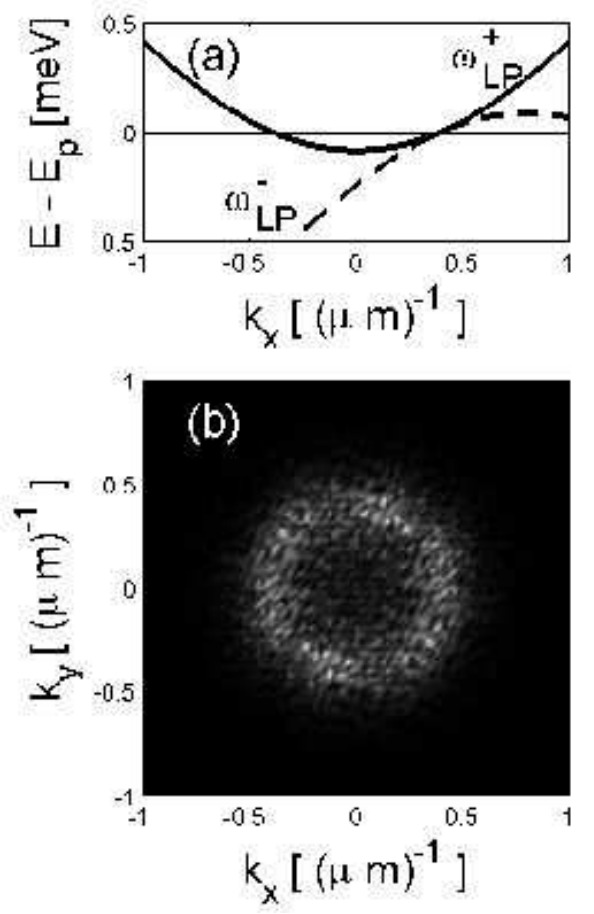

FIG. 12: Energy dispersion of the LP branches in the weak excitation regime.(b) Intensity (arb. units) of the photonic resonant Rayleigh scattering signal $\left|\delta \tilde{\phi}_{C}(\mathbf{k})\right|^{2}$. Excitation parameters: $k_{p}=0.4 \mu \mathrm{m}^{-1}$ (along the x-axis), $\hbar \omega_{p}-\hbar \omega_{L P}\left(\mathbf{k}_{p}\right)=0 \mathrm{meV}, \hbar g\left|\Psi_{X}^{s s}\right|^{2}=0.0001 \mathrm{meV}$. The photonic and exciton potentials are those shown in Fig [1] The speckles of the elastic RRS ring are due to the disordered excitonic potential. The chosen plot range is such that a few speckles saturate the gray scale.

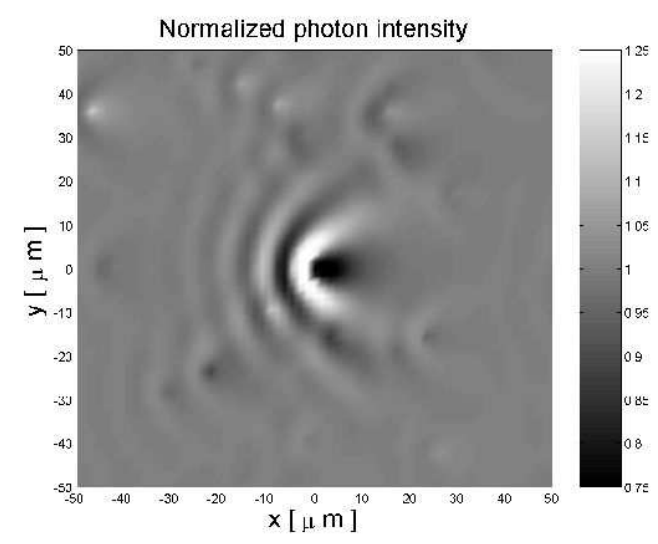

FIG. 13: Spatial profile of the normalized cavity photon density, i.e., $I_{C}(\mathbf{x}) / I_{C}^{\text {hom }}$. Excitation parameters and potentials as in Fig. 12 The coherent diffusion pattern induced by the point defect at the origin is the main feature on top of the random landscape produced by the exciton disorder. 

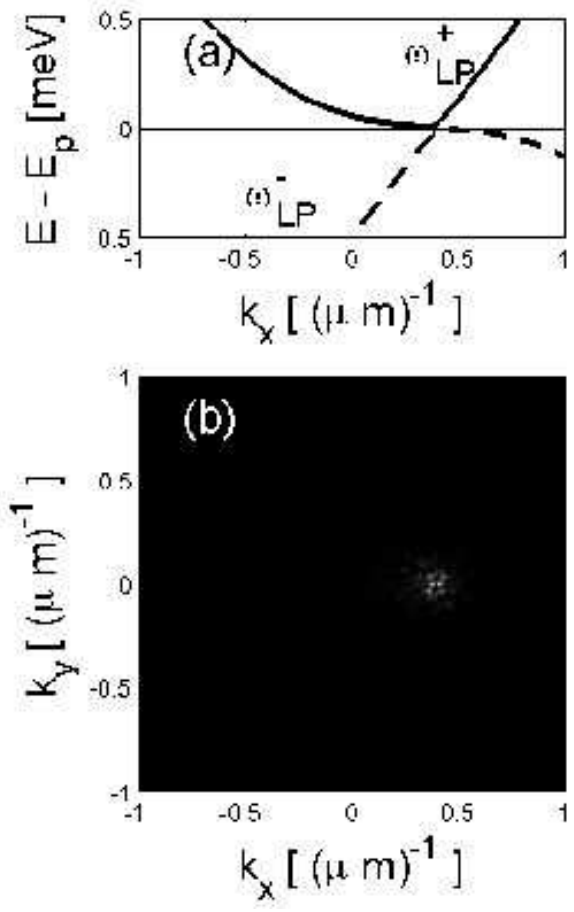

FIG. 14: Superfluid regime. Same parameters as in Fig. 12 but with $\hbar \omega_{p}-\hbar \omega_{L P}\left(\mathbf{k}_{p}\right)=0.467 \mathrm{meV}, \hbar g\left|\Psi_{X}^{s s}\right|^{2}=1 \mathrm{meV}$. In this superfluid regime, the RRS elastic ring has collapsed.

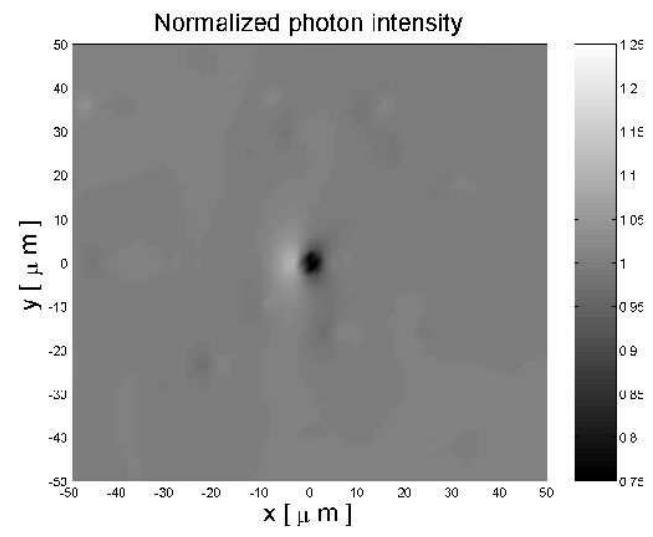

FIG. 15: Spatial profile of the normalized cavity photon density in the superfluid regime. Excitation parameters as in Fig. 14] To compare with the normal (weak) excitation regime, see Fig. 13

Precisely, we have plotted the normalized quantity

$$
\frac{I_{C}(\mathbf{x})}{I_{C}^{h o m}}=\frac{\left|\phi_{C}^{s s} e^{i \mathbf{k}_{p} \mathbf{x}}+\delta \phi_{C}(\mathbf{x})\right|^{2}}{\left|\phi_{C}^{s s}\right|^{2}}
$$

i.e., the total photon field intensity (homogeneous solution + potential-induced perturbation) normalized to the intensity of the homogeneous solution without the poten- tial. For the considered potentials in Fig. 11 the dominant feature is due to the photonic point defect. The polariton plane wave driven by the pump is coherently scattered by the photonic defect (located at the position $x=y=0$ ), producing a peculiar interference pattern, characterized by parabolic wavefronts. In fact, the polariton field scattered by the point defect is a cylindrical wave. Hence, if we consider only one defect and $\mathbf{k}_{p}$ is along the $\mathrm{x}$-direction, the total field has the form $f(\mathbf{x})=e^{i k_{p} x}+\beta e^{i k_{p} \sqrt{x^{2}+y^{2}}}$. The constant phase curves are given by the condition $k_{p} x+k_{p} \sqrt{x^{2}+y^{2}}=2 \pi n$, whose solutions describe parabolic wavefronts with a symmetry axis oriented along the x-direction, as nicely depicted by the exact solution in Fig. [13. Due to the presence of the exciton potential, additional disordered features are superimposed on the main interference pattern produced by the photonic point defect.

\section{B. Superfluid regime}

In presence of interactions, we have seen that the spectrum of polariton Bogoliubov excitations is dramatically different from the unperturbed case. This manifestation of polariton many-body physics can be probed in a sensitive way by the resonant Rayleigh scattering emission. In Fig. 14, we start by considering the most spectacular regime of polariton superfluidity. This regime can be achieved when the pump is resonant with the interactionrenormalized polariton dispersion at the pump wavevector $\left(\Delta_{p}=0\right)$ and when the sound velocity $c_{s}$ [see Eq. (21)] of the interacting polariton fluid is larger than the flow velocity $v_{p}=\hbar k_{p} / m_{L P}$ imprinted by the pump beam. This situation is more favorable to obtain for excitation close to the bottom of the LP dispersion, implying smaller pump flow velocity $v_{p}$ and smaller excitation density necessary to have $c_{s}>v_{p}$. As depicted by Fig. 14(a), in this case, the equation $\Re\left[\omega_{L P}^{ \pm}(\mathbf{k})\right]-\omega_{p}=0$ has no solutions for $\mathbf{k} \neq \mathbf{k}_{p}$, meaning that no final states are available for the elastic scattering induced by the static potential. As a dramatic consequence, the elastic ring in Fig. 12(b) collapses. As shown in Fig. 14(b), only a weak emission around the pump wavevector $\mathbf{k}_{p}$ is left, due to non-resonant processes, which are allowed by the finite broadening of the polariton modes.

The real space pattern is shown in Fig. [15] showing that the effect of the disorder remains localized around the defect positions. Hence, the polaritonic propagation is superfluid. In analogous way to liquid Helium and atomic condensates 1, 2], we can state that the polariton fluid has a superfluid behaviour according to the Landau criterion, if and only if both following conditions are satisfied

(a) $\omega_{L P, U P}^{+}(\mathbf{k})>\omega_{p}$ for every $\mathbf{k} \neq \mathbf{k}_{p}$.

(b) $\omega_{p}>\omega_{L P}(0)$, i.e. there is an elastic ring in the weak excitation regime.

We point out that the condition (b) is necessary to 
have a meaningful definition of polariton superfluidity. In fact, if $\omega_{p}<\omega_{L P}(0)$, already in the weak excitation regime there are no real states at the pump energy and there is no resonant Rayleigh scattering elastic ring. Note that the conditions (a) and (b) are achieved not only in the resonant case $\Delta_{p}=0$. Within the parabolic approximation in Eq. (18), conditions (a) and (b) are satisfied when $\Delta_{p} \leq 0$ and

$$
-g_{L P}\left|\psi_{L P}^{s s}\right|^{2}-\frac{m_{L P} v_{p}^{2}}{2 \hbar}<\Delta_{p}<g_{L P}\left|\psi_{L P}^{s s}\right|^{2}-\frac{m_{L P} v_{p}^{2}}{\hbar} .
$$

The calculations here reported show the robustness of the superfluid flow with respect to elastic processes, such as the scattering on static defects. As the Landau criterion for superfluidity involves also inelastic processes (e.g., emission of crystal phonons or heating of residual free carriers), it is important to note that whenever the superfluidity condition (a) for elastic scattering is fulfilled, then it is satisfied a fortiori also for the inelastic channels. In fact, the stability of the mean-field solution implies that almost no Bogoliubov quasi-particles are present above a stable mean-field solution, so whenever $\omega_{L P}^{+}(\mathbf{k})>\omega_{p}$, no final states are available for the polariton system to lower its energy by transferring energy to its environment. This is here assumed to be at almost zero temperature, so that it can only absorb energy from the polariton system. Friction is therefore absent in this regime.

On the other hand, at a finite temperature of the crystal a thermally excited "normal" component can appear in the polariton fluid because of its heating due the interaction with the phonon bath and the residual free carriers. As it happens in liquid Helium, this normal component experiences a finite friction when flowing onto the defect. However, its magnitude vanishes at low temperatures, and in any case it does not affect the superfluidity of the co-existing superfluid component in a two-fluid picture.

\section{Precursors of parametric instabilities and branch sticking}

In the case $\Delta_{p}>0$ (i.e., pump frequency higher than the renormalized frequency of the pumped mode), the resonant Rayleigh scattering response is completely different, as shown in Fig. 16 and 18 In this regime, the two LP branches stick together, while there is a splitting of their imaginary parts [see, e.g., the analogous situation in Fig. 8 and Fig. 9. Such a scenario represents the precursor of a multi-mode parametric instability, which can be triggered by further increasing the excitation density. In contrast to the superfluid regime, a deformed RRS ring is apparent in Fig. 16(b) and Fig. 18(b). In particular, depending on the topology of the $\mathbf{k}$-space region where the LP branches stick, the RRS intensity is strongly amplified either on a segment parallel to $y$ including the point $\mathbf{k}_{p}$ [Fig [16(b)], or around two points of
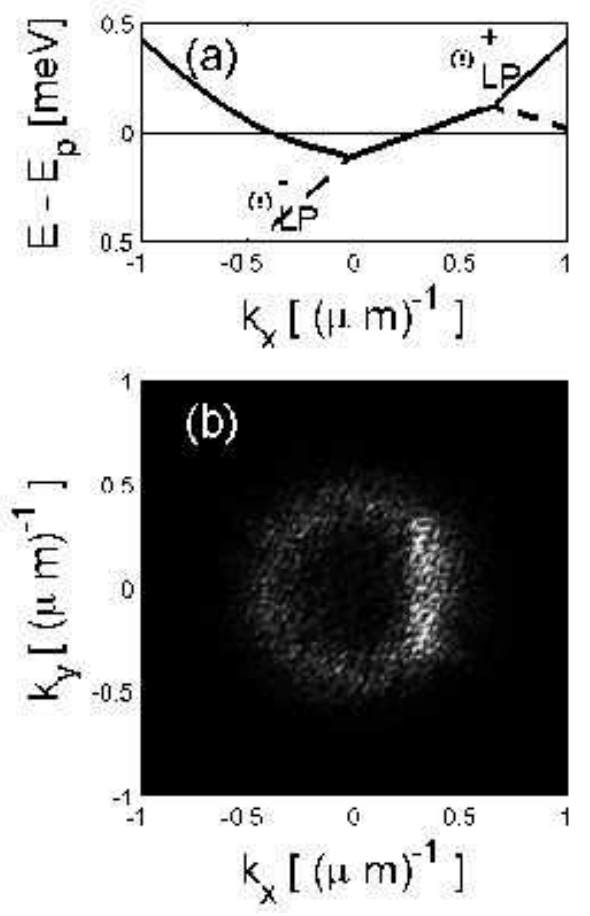

FIG. 16: Same parameters as in Fig. 12 but with $\hbar \omega_{p}-$ $\hbar \omega_{L P}\left(\mathbf{k}_{p}\right)=0.1 \mathrm{meV}, \hbar g\left|\Psi_{X}^{s s}\right|^{2}=0.07 \mathrm{meV}$. Branch sticking and amplified RRS are precursors of a parametric instability.

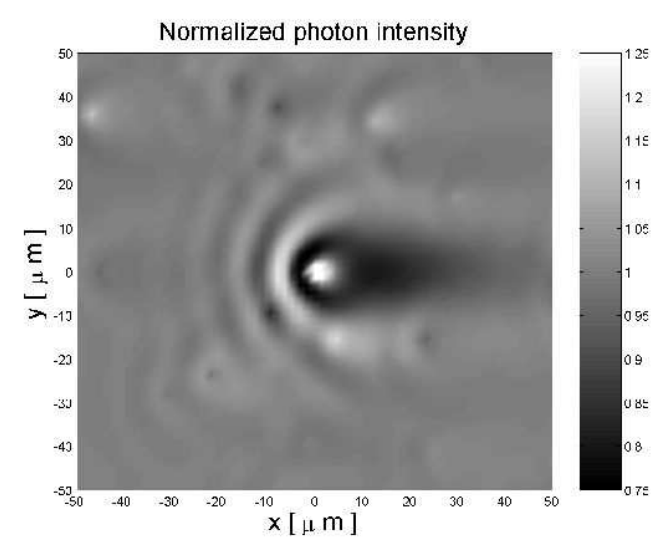

FIG. 17: Spatial profile of the normalized cavity photon density. Excitation parameters as in Fig. 16] Note that the gray scale of this plot is saturated in the region around the point defect at the origin.

the straight line parallel to $y$ and passing through the point $\mathbf{k}_{p}$ [Fig. [18(b)]. The main consequence of this in the real-space pattern of Fig. 17 is an overall amplification of the density modulation induced by the defect, in stark contrast with the superfluid regime. In particular, note the long "shadow" in the downstream direction with respect to the central defect, which extends to relatively 

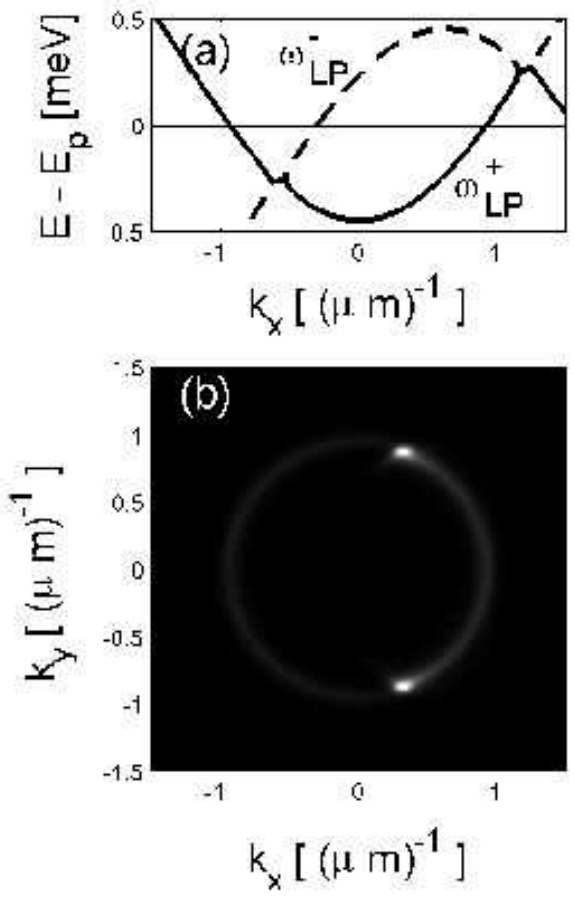

FIG. 18: Excitation parameters: $k_{p}=0.314 \mu \mathrm{m}^{-1}$ (along the x-axis), $\hbar \omega_{p}-\hbar \omega_{L P}\left(\mathbf{k}_{p}\right)=0.47 \mathrm{meV}, \hbar g\left|\Psi_{X}^{s s}\right|^{2}=0.075 \mathrm{meV}$. Note that the gray scale of this plot is saturated around the two brightest points. Here, we consider the situation of a dominant photonic potential $\left(V_{X}=0\right)$.

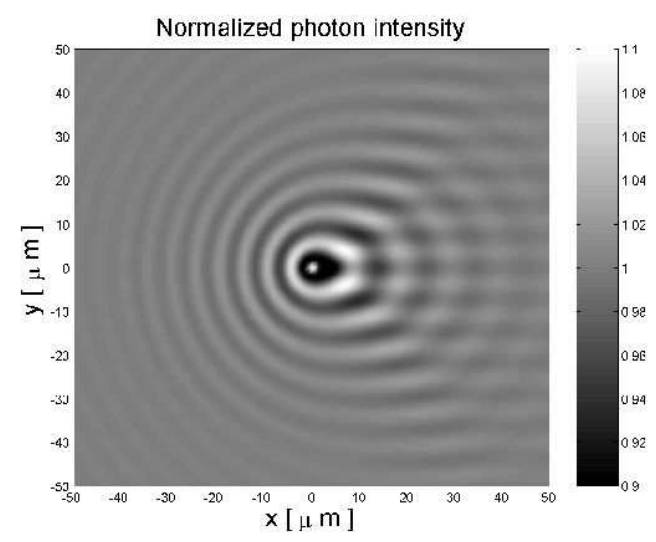

FIG. 19: Spatial profile of the normalized cavity photon density. Same parameters as in Fig. 18

far distances thanks to the linewidth narrowing effect. In Fig [19] the shadow of the defect is even more peculiar, showing a series of fringes parallel to the $x$ direction. These can be explained in terms of the interference between the pump and the two peaks in $\mathbf{k}$-space shown in Fig 18(b).
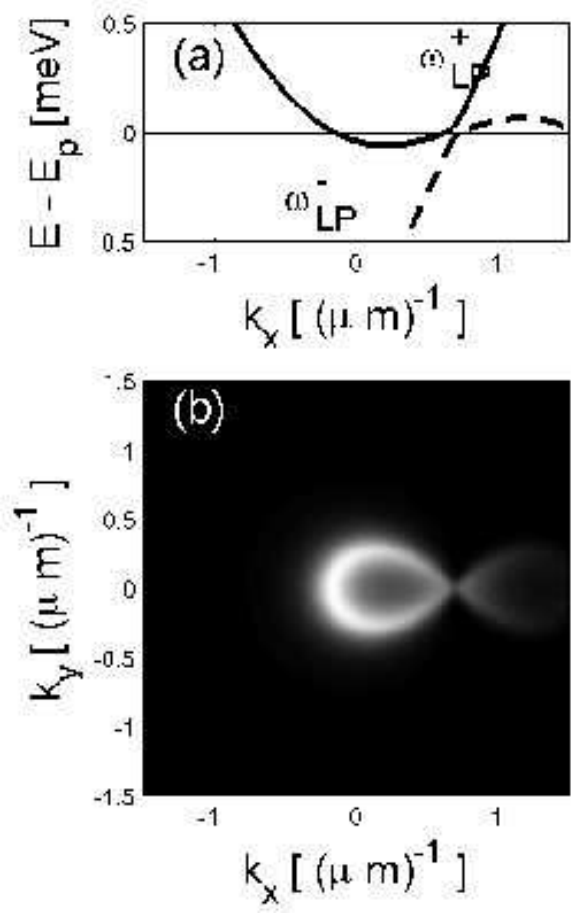

FIG. 20: Cherenkov regime. Parameters: $\omega_{X}=\omega_{C}(0)-$ $1 \mathrm{meV}, k_{p}=0.7 \mu \mathrm{m}^{-1}$ (along the x-axis), $\hbar \omega_{p}-\hbar \omega_{L P}\left(\mathbf{k}_{p}\right)=$ $0.599 \mathrm{meV}, \hbar g\left|\Psi_{X}^{s s}\right|^{2}=1 \mathrm{meV}$. Here, we consider the situation of a dominant photonic potential $\left(V_{X}=0\right)$.

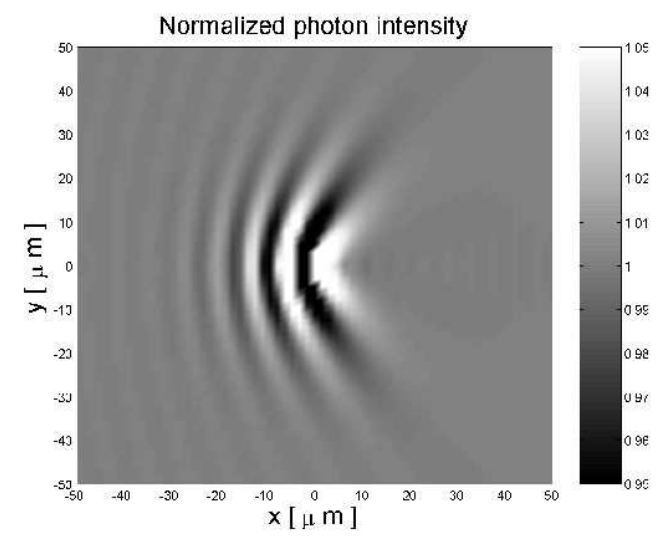

FIG. 21: Spatial profile of the normalized cavity photon density. Same parameters as in Fig. [20] The photonic point defect produces Cherenkov-like wavefronts. Note that, in order to show the peculiar shape of the wavefronts, the gray scale of this plot is saturated in the region around the point defect.

\section{Cherenkov regime}

Here, we consider the opposite case $\Delta_{p} \leq 0$, but with an excitation density which is not high enough to enter the superfluid regime characterized by the condition in 

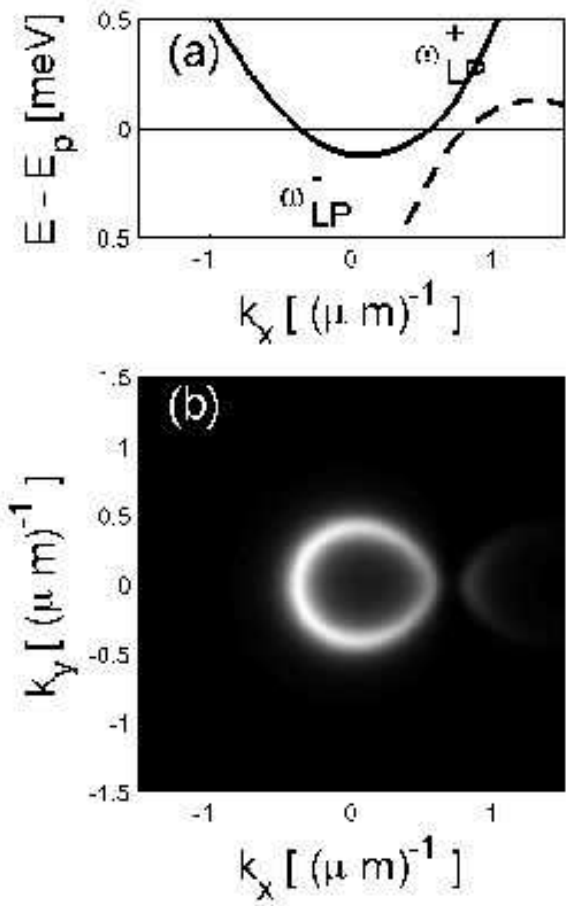

FIG. 22: Parameters: $\omega_{X}=\omega_{C}(0), k_{p}=0.7 \mu \mathrm{m}^{-1}$ (along the X-axis), $\hbar \omega_{p}-\hbar \omega_{L P}\left(\mathbf{k}_{p}\right)=0.3 \mathrm{meV}, \hbar g\left|\Psi_{X}^{s s}\right|^{2}=0.6 \mathrm{meV}$.

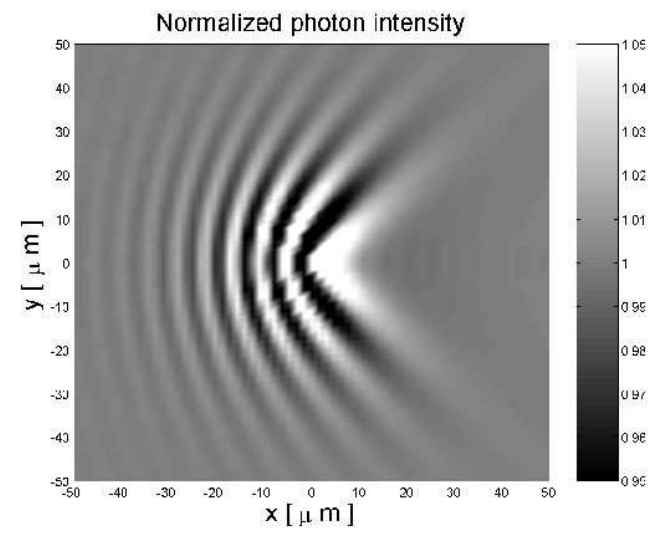

FIG. 23: Spatial profile of the normalized cavity photon density. Same parameters as in Fig. 22 The gray scale of this plot is saturated in the region around the point defect.

Eq. (27). For the sake of clarity, as we have already done for Fig. [18] and Fig. [19] we take here $V_{X}=0$, so to concentrate on the effect of a single defect. This situation can be realistically realized, e.g., when there is a single photonic defect (natural or artificial) which is dominant over the background excitonic disorder. In Fig. 201 (a), we consider the resonant case $\left(\Delta_{p}=0\right)$, with a polariton sound speed $c_{s}<v_{p}$. As shown in Fig. 20 (b) the weak excitation elastic RRS ring is replaced by an asym-
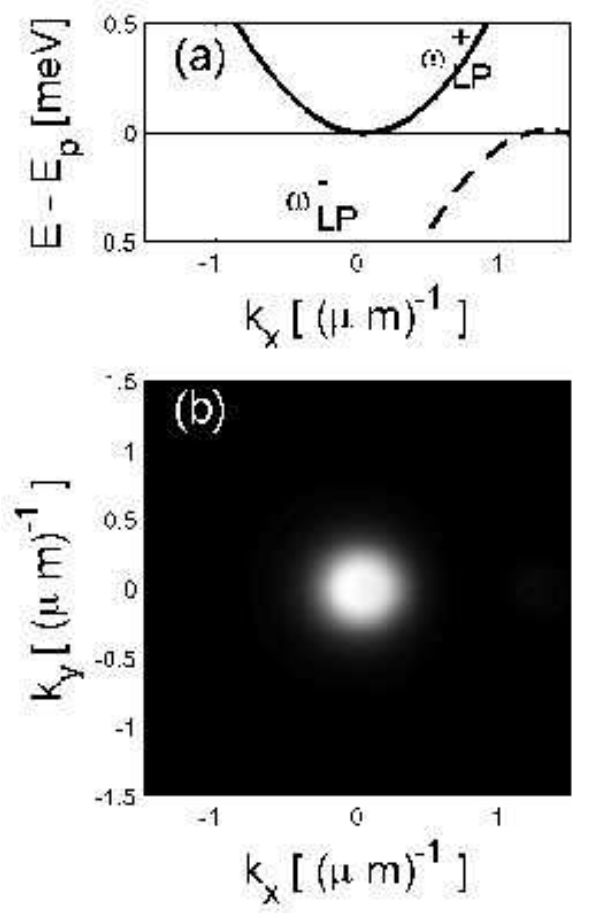

FIG. 24: Parameters: $\omega_{X}=\omega_{C}(0), k_{p}=0.7 \mu \mathrm{m}^{-1}$ (along the $\mathrm{x}$-axis), $\hbar \omega_{p}-\hbar \omega_{L P}\left(\mathbf{k}_{p}\right)=0.2 \mathrm{meV}, \hbar g\left|\Psi_{X}^{s s}\right|^{2}=0.6 \mathrm{meV}$.

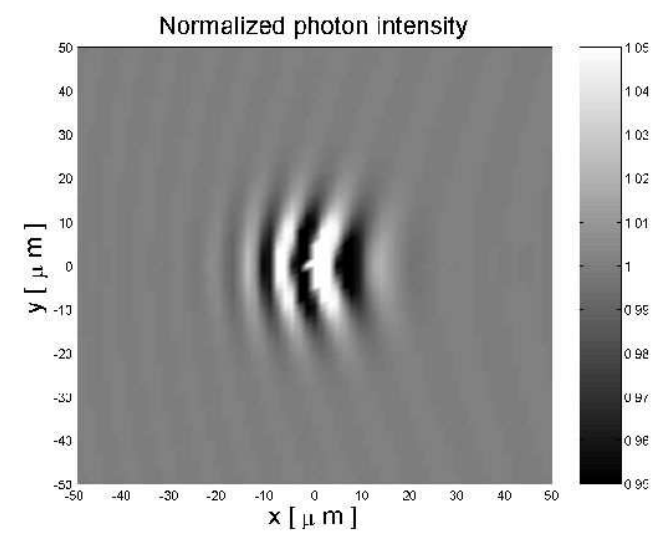

FIG. 25: Spatial profile of the normalized cavity photon density. Same parameters as in Fig. 24] The gray scale of this plot is saturated in the region around the point defect.

metric pattern, which is strongly deformed and shows a singularity at the pump wavevector. The aperture angle $2 \theta$ of the singularity at $\mathbf{k}_{p}$ satisfies the simple condition $\cos \theta=c_{s} / v_{p}$. In this $c_{s}<v_{p}$ regime where the polariton fluid is moving at a supersonic speed, the defect produces a peculiar real-space pattern (Fig. 21) showing linear Cherenkov-like wavefronts [39, 40]. The aperture $2 \phi$ of the Cherenkov angle has the usual value $\sin \phi=c_{s} / v_{p}$. This behavior is easily understood from a physical stand- 
point as follows: a moving fluid propagating along the positive $x$ direction in the presence of a static defect is equivalent, under a Galilean transformation, to a defect moving in the negative $x$ direction in a fluid at rest. This situation is a familiar one, as it corresponds to the wavefronts created by a moving duck on the surface of a lake. The rounded region on the left-hand side of the $\mathbf{k}$-space pattern in Fig. 20(b) was not present in the standard theory of Cherenkov emission in non-dispersive media 39] and it is due to the fact that the Bogoliubov dispersion is linear only in the neighborhood of $\mathbf{k}_{p}$ and then it bends upwards. A remarkable consequence of this property is the oscillatory perturbation shown by the real-space pattern upstream with respect to the defect, as apparent in Fig 21

In the case $\Delta_{p}<0$, the branches $\omega_{L P}^{+}$and $\omega_{L P}^{-}$are disconnected, as well as the corresponding RRS emission pattern, as depicted in Fig. 222 The real-space wavefronts shown in Fig. 23 are still Cherenkov-like, since the separation between the two branches is relatively small.

The situation of Fig. 24] and 25 is instead different, with a full gap opened between the two LP branches. The $\mathbf{k}$-space emission is then concentrated around the point $\mathbf{k}_{a}$ where the Bogoliubov dispersion touches the line $\omega=\omega_{p}$. Correspondingly, the near-field pattern shows a localized perturbation around the defect, with a peculiar stripe pattern. This pattern is due to the interference of the Rayleigh scattering at $\mathbf{k}_{a}$ and the pump beam at $\mathbf{k}_{p}$, so that the wavevector corresponding to the fringes is equal to $\mathbf{k}_{p}-\mathbf{k}_{a}$.

\section{CONCLUSIONS}

In conclusion, in this Festschrift paper, we have presented a comprehensive analysis of the exotic collective excitations of a moving polariton fluid driven by a continuous-wave optical pump, which were recently predicted in a short Letter 27]. We have analyzed in detail the interplay between the non-trivial dispersions of the polariton Bogoliubov excitations and the onset of singlemode (Kerr) or multi-mode (parametric) instabilities. We have studied the propagation of the polariton fluid in presence of static perturbation potential acting both on the photonic and exciton component of the polariton field. In particular, we have shown the strict connection between the dispersion of the elementary excitations in a quantum fluid of microcavity polaritons and the intensity and shape of the resonant Rayleigh scattering on defects. We have pointed out some experimentally accessible consequences of polaritonic superfluidity for realistic microcavity parameters. In addition, we have shown that in the present non-equilibrium system, it is possible to have spectra of collective excitations, which are not accessible in systems close to the thermal equilibrium, such as superfluid helium or the ultracold atomic condensates. These spectra of excitations can be dramatically changed by tuning the pump excitation parameters, namely its frequency, incidence angle and intensity. We have shown a very rich phenomenology in the far-field and near-field images of the resonant Rayleigh scattering emission.

\section{ACKNOWLEDGMENTS}

LPA-ENS is a "Unité de Recherche de l'Ecole Normale Supérieure et des Universités Paris 6 et 7, associées au CNRS". CC wishes to thank all the authors contributing to the present Festschrift volume for many stimulating discussions on the physics of semiconductor microcavities. IC acknowledges stimulating discussions with C. Tozzo and F. Dalfovo on the subject of modulational instabilities in quantum fluids.
[1] D. Pines and P. Nozieres, The theory of quantum liquids Vols.1 and 2 (Addison-Wesley, Redwood City, 1966).

[2] L. Pitaevskii and S. Stringari, Bose-Einstein condensation (Oxford University Press, 2003).

[3] A. J. Leggett, Rev. Mod. Phys. 71, S318-S323 (1999).

[4] C. Weisbuch, M. Nishioka, A. Ishikawa, and Y. Arakawa, Phys. Rev. Lett. 69, 3314 (1992).

[5] For a recent review, see Semicond. Sci. Technol. 18, Special Issue on Microcavities, Guest Editors J. Baumberg and L. Viña, Publisher S. Quin (Bristol, UK, 2003).

[6] P. G. Savvidis, J. J. Baumberg, R. M. Stevenson, M. S. Skolnick, D. M. Whittaker, J. S. Roberts, Phys. Rev. Lett. 84, 1547 (2000).

[7] C. Ciuti, P. Schwendimann, B. Deveaud, and A. Quattropani, Phys. Rev. B 62, R4825 (2000).

[8] M. Saba, C. Ciuti, J. Bloch, V. Thierry-Mieg, R. André, Le Si Dang, S. Kundermann, A. Mura, G. Bongiovanni, J. L. Staehli, B. Deveaud, Nature (London) 414, 731 (2001).
[9] P. G. Savvidis, C. Ciuti, J. J. Baumberg, D. M. Whittaker, M. S. Skolnick, and J. S. Roberts Phys. Rev. B 64, 075311 (2001)

[10] A. Huynh, J. Tignon, O. Larsson, Ph. Roussignol, C. Delalande, R. André, R. Romestain, Le Si Dang, Phys. Rev. Lett. 90, 106401 (2003).

[11] S. Kundermann, M. Saba, C. Ciuti, T. Guillet, U. Oesterle, J. L. Staehli, and B. Deveaud, Phys. Rev. Lett. 91, 107402 (2003).

[12] J. J. Baumberg, P. G. Savvidis, R. M. Stevenson, A. I. Tartakovskii, M. S. Skolnick, D. M. Whittaker, and J. S. Roberts, Phys. Rev. B 62, R16247 (2000).

[13] R. M. Stevenson, V. N. Astratov, M. S. Skolnick, D. M. Whittaker, M. Emam-Ismail, A. I. Tartakovskii, P. G. Savvidis, J. J. Baumberg, and J. S. Roberts, Phys. Rev. Lett. 85, 3680 (2000).

[14] R. Houdré, C. Weisbuch, R. P. Stanley, U. Oesterle, and M. Ilegems, Phys. Rev. Lett. 85, 2793 (2000).

[15] D. M. Whittaker, Phys. Rev. B 63, 193305 (2001) 
[16] G. Messin, J. Ph. Karr, A. Baas, G. Khitrova, R. Houdré, R. P. Stanley, U. Oesterle, E. Giacobino, Phys. Rev. Lett. 87, 127403 (2001).

[17] G. Dasbach, M. Schwab, M. Bayer, and A. Forchel, Phys. Rev. B 64, 201309 (2001).

[18] G. Dasbach, M. Schwab, M. Bayer, D.N. Krizhanovskii, A. Forchel, Phys. Rev. B 66, 201201 (2002).

[19] C. Ciuti, P. Schwendimann, A. Quattropani, Phys. Rev. B 63, 041303(2001).

[20] P. Schwendimann, C. Ciuti, A. Quattropani, Phys. Rev. B 68, 165324 (2003).

[21] J. Ph. Karr, A. Baas, R. Houdré, Elisabeth Giacobino, Phys. Rev. A 69, 031802 (2004).

[22] J. Ph. Karr, A. Baas, and E. Giacobino, Phys. Rev. A 69, 063807 (2004).

[23] C. Ciuti, Phys. Rev. B 64, 245304 (2004).

[24] W. Langbein, Proc. 26th Int. Conf. on the Physics of Semiconductors; ICPS 26 (Edinburgh, UK, 2002).

[25] W. Langbein, Phys. Rev. B 70, 205301(2004).

[26] S. Savasta, O. Di Stefano, V. Savona, W. Langbein, cond-mat/0411314

[27] I. Carusotto and C. Ciuti, Phys. Rev. Lett. 93, 166401 (2004).

[28] H. Stolz, D. Schwarze, W. von der Osten, and G. Weimann, Phys. Rev. B 47, 9669 (1993).

[29] R. Houdré, C. Weisbuch, R. P. Stanley, U. Oesterle, and
M. Ilegems, Phys. Rev. B 61, R13333 (2000).

[30] W. Langbein and J. M. Hvam, Phys. Rev. Lett. 88, 047401 (2002), and references therein.

[31] C. Ciuti, P. Schwendimann, and A. Quattropani, Semicond. Sci. Technol. 18, S279-S293 (2003).

[32] S. Savasta, O. Di Stefano, and R. Girlanda, Phys. Rev. Lett. 90, 096403 (2003).

[33] B. Wu and Q. Niu, Phys. Rev. A 64, 061603(R) (2001).

[34] A. Baas, J. Ph. Karr, H. Eleuch, and E. Giacobino, Phys. Rev. A 69, 023809 (2004).

[35] N. A. Gippius, S. G. Tikhodeev, V. D. Kulakovskii, D. N. Krizhanovskii, A. I. Tartakovskii, Europhys. Lett. 67, 997 (2004).

[36] A. Baas, J. Ph. Karr, M. Romanelli, A. Bramati, and E. Giacobino, Phys. Rev. B 70, 161307 (R) (2004).

[37] R. W. Boyd, Nonlinear Optics (Academic Press, London, 1992).

[38] C. Ciuti, P. Schwendimann, A. Quattropani, Phys. Rev. B 63, 041303(R) (2001); D. M. Whittaker, Phys. Rev. B 63, 193305 (2001).

[39] J. V. Jelley, Cerenkov radiation and its applications, Pergamon Press, 1958.

[40] I. Carusotto, M. Artoni, G. C. La Rocca, F. Bassani, Phys. Rev. Lett. 87, 064801 (2001). 